\title{
Equivariant-bivariant Chern character for profinite groups
}

\author{
by P. Baum and P. Schneider
}

In this paper we propose a definition of (delocalized) equivariant-bivariant cohomology. The coefficient ring over which we work will be the complex numbers $\mathbb{C}$. However, the cyclotomic field $\widetilde{\mathbb{Q}}$ (i.e. the field obtained by adjoining all roots of unity to $\mathbb{Q}$ ) could also have been used. Throughout, $G$ will denote a locally compact, totally disconnected topological group. We let $Y$ and $X$ be locally compact Hausdorff topological spaces with given continuous actions $G \times Y \longrightarrow Y$ and $G \times X \longrightarrow X$. In this context, we define our equivariant bivariant cohomology, denoted $H_{G, c}^{*}(Y, X)$. This is a $\mathbb{Z}$-graded theory. Each $H_{G, c}^{n}(Y, X)$, for $n \in \mathbb{Z}$, is a vector space over $\mathbb{C}$.

For most of the paper we shall assume: (1) $Y$ admits a triangulation as a locally finite simplicial complex, such that each $g \in G$ acts as a simplicial map. (2) The action of $G$ on $Y$ is proper and smooth, i.e., the stabilizer of each simplex is compact open. (3) The quotient space $G \backslash Y$ is compact.

A typical example is a reductive $p$-adic group acting on its affine Bruhat-Tits building. For the action of $G$ on $X$, no assumption is made other than that $X$ is a locally compact Hausdorff topological space, and the action of $G$ on $X$ is continuous. As usual, $C_{0}(X)$ is the abelian $C^{*}$-algebra of all continuous, complex valued, vanishing-at-infinity functions on $X$, and $K K_{G}^{*}\left(C_{0}(Y), C_{0}(X)\right)$ is Kasparov equivariant bivariant $K$-theory. Granted conditions (1)-(3) we conjecture that there is a Chern character

$$
c h: K K_{G}^{j}\left(C_{0}(Y), C_{0}(X)\right) \longrightarrow \bigoplus_{n \in \mathbf{Z}} H_{G, c}^{j+2 n}(Y, X), j=0,1
$$

which becomes an isomorphism of $\mathbb{C}$-vector spaces when $K K_{G}^{j}\left(C_{0}(Y), C_{0}(X)\right)$ is tensored (over $\mathbb{Z}$ ) with $\mathbb{C}$.

This conjecture is proved for $G$ profinite in $\S 2-\S 4$. In constructing the Chern character (for profinite $G$ ) a bivariant version of Segal localization [Seg] is used. $\S 1$ is devoted to defining $H_{G, c}^{*}(Y, X)$ (here, in fact, any field of characteristic zero could be used as the field of coefficients) and to showing that the definition unifies examples studied by various authors. $\S 5$ proves a homotopy theoretic representability theorem for $H_{G, c}^{*}(p t, X)$. In an appendix we make precise some properties of the Dold-Kan correspondence which are used in proving the representability theorem.

Remark. Conditions (1)-(3) might seem somewhat restrictive. To test this point of view, consider the case when $Y$ is a compact Hausdorff topological space, $G$ 
is the trivial one-element group, and $X$ is a point. $K K^{*}(C(Y), \mathbb{C})$ is then the Kasparov $K$-homology of $Y$. If $Y$ is a finite simplicial complex, there is a Chern character

$$
\operatorname{ch}: K K^{j}(C(Y), \mathbb{C}) \longrightarrow \bigoplus_{n \in \mathbf{Z}} H_{j+2 n}(Y ; \mathbb{Q}), j=0,1
$$

which becomes an isomorphism of $\mathbb{Q}$-vector spaces when $K K^{j}(C(Y), \mathbb{C})$ is tensored (over $\mathbb{Z}$ ) with $\mathbb{Q}$. But the case when $Y$ is a compact Hausdorff topological space appears to be unsolved at the present time, i.e. there seems to be no homology theory (defined by purely topological methods) on the category of compact Hausdorff topological spaces which is the target of a Chern character isomorphism from $K K^{j}(C(Y), \mathbb{C}) \otimes \mathbb{C}$. In view of this, conditions (1)-(3) may, in fact, be quite appropriate.

Our Chern character conjecture is proved elsewhere for $G$ discrete.

Terminology. Following Bourbaki [B-GT] locally compact topological spaces will be assumed to be Hausdorff.

We thank A.K.Bousfield, B. Gray, R. Jardine, M. Mandell, and J.P. May for helpful and enlightening discussions.

P. Baum was partially supported by an NSF grant.

\section{The construction of bivariant cohomology and special cases}

Let $G$ be a locally compact and totally disconnected group. An element $g \in G$ is called compact if it is contained in a compact subgroup. We always make the assumption that the subset $G_{0}$ of all compact elements is locally closed in $G$. Let $X$ be a $G$-space by which we always mean a locally compact space with a continuous $G$-action. We refer to [Sch] for the notion of a $G$-equivariant sheaf $F$ (of $\mathbb{C}$-vector spaces) on $X$ and for a construction of the basic operations on such sheaves. Recall that a $G$-equivariant sheaf on the point is a smooth $G$ - $\mathbb{C}$-module.

Of basic importance for us will be the space

$$
\hat{X}:=\{(g, x) \in G \times X: g \text { compact and } g x=x\}
$$

equipped with the subspace topology in $G \times X$. It again is a $G$-space via

$$
g(h, x):=\left(g h g^{-1}, g x\right) .
$$

Let

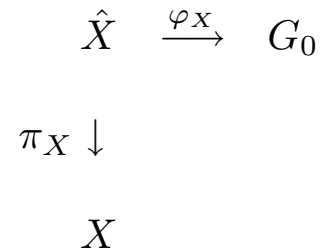


denote the obvious projection maps. Also let $Y$ be a second $G$-space. We will call the hyperext-groups

$$
H_{G, c}^{*}(Y, X):=\operatorname{Ext}_{\mathrm{Sh}_{G}\left(G_{0}\right)}^{*}\left(R \varphi_{Y !} \mathbb{C}, R \varphi_{X !} \mathbb{C}\right)
$$

for the abelian category $\operatorname{Sh}_{G}\left(G_{0}\right)$ of $G$-equivariant sheaves on $G_{0}$ the (compactly) delocalized equivariant bivariant cohomology theory of $(Y, X)$. Since the functor $X \longmapsto \hat{X}$ respects proper $G$-maps this bivariant theory is covariant for proper $G$-maps in $Y$ and is contravariant for proper $G$-maps in $X$.

There is a somewhat more down to earth reformulation of the above definition in terms of modules instead of sheaves. This rests on the following fact. Let $Z$ be any locally compact and totally disconnected space and let $\Gamma_{c}(Z, \mathbb{C})$ be the ring of $\mathbb{C}$-valued locally constant functions with compact support on $Z$. A module $M$ for this ring (which in general has no unit) is called nondegenerate if $\Gamma_{c}(Z, \mathbb{C}) \cdot M=$ $M$. Let $\operatorname{Mod}\left(\Gamma_{c}(Z, \mathbb{C})\right)$ denote the abelian category of nondegenerate $\Gamma_{c}(Z, \mathbb{C})$ modules. Then the functor of taking global sections with compact support

$$
\Gamma_{c}(Z, .): \operatorname{Sh}(Z) \stackrel{\sim}{\longrightarrow} \operatorname{Mod}\left(\Gamma_{c}(Z, \mathbb{C})\right)
$$

is an equivalence of categories ([BZ] 1.14). Given any nondegenerate $\Gamma_{c}(Z, \mathbb{C})$ module $M$ the sections of the corresponding sheaf over a compact open subset $U \subseteq Z$ are given by $\Gamma(U, \mathbb{C}) \underset{\Gamma_{c}(Z, \mathbb{C})}{\otimes} M$. Suppose now that $Z$ in addition is a $G$ space. Then $\Gamma_{c}(Z, \mathbb{C})$ is a smooth $G$-module. We let $\operatorname{Mod}\left(\Gamma_{c}(Z, \mathbb{C}), G\right)$ denote the category of smooth $\left(\Gamma_{c}(Z, \mathbb{C}), G\right)$-modules, i.e., nondegenerate $\Gamma_{c}(Z, \mathbb{C})$-modules $M$ which carry a smooth $G$-action such that the compatibility condition

$$
g(f \cdot m)=(g f) \cdot(g m) \quad \text { for any } g \in G, f \in \Gamma_{c}(Z, \mathbb{C}), \text { and } m \in M
$$

is satisfied. It is easy to see that the functor $\Gamma_{c}(Z,$.$) also induces an equivalence$ of categories

$$
\operatorname{Sh}_{G}(Z) \stackrel{\sim}{\longrightarrow} \operatorname{Mod}\left(\Gamma_{c}(Z, \mathbb{C}), G\right) .
$$

Using this we may rewrite the definition of our bivariant cohomology theory as the hyperext-groups

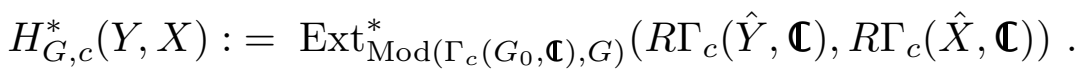

in the module category $\operatorname{Mod}\left(\Gamma_{c}\left(G_{0}, \mathbb{C}\right), G\right)$.

We also want to introduce a version with $G$-compact supports of our theory where we allow in the first variable an arbitrary (not necessarily locally compact) Hausdorff space $Z$ with a proper $G$-action ([B-GT] III $\S 4.1$ ). Following [BCH] we call $Z G$-compact if $G \backslash Z$ is compact. Note that any $G$-compact $Z$ necessarily is locally compact ([B-GT] III $\S 4$ Ex. 10.e). Hence we may define

$$
H_{G, !}^{*}(Z, X):=\lim _{Y \subseteq Z} H_{G, c}^{*}(Y, X)
$$


where $Y$ runs over all $G$-invariant and $G$-compact (closed) subsets of $Z$.

Let us look at various special cases in order to see that this new concept generalizes and unifies a number of constructions which exist in the literature.

A. Assume $Y=p t$ to be a point. We get the equivariant cohomology groups

$$
H_{G, c}^{*}(p t, X)=H^{*}\left(G_{0}, G ; R \varphi_{X !} \mathbb{C}\right)
$$

In addition assume $G$ to be compact. Then $G$ has no higher group cohomology in smooth $G$ - $\mathbb{C}$-modules. It follows that

$$
H_{G, c}^{*}(p t, X)=H_{c}^{*}(\hat{X}, \mathbb{C})^{G}
$$

is the subspace of $G$-fixed vectors in the cohomology with compact support of $\hat{X}$. Moreover the quotient map $q: \hat{X} \longrightarrow G \backslash \hat{X}$ is a proper map between locally compact spaces and has compact and totally disconnected fibers. Hence a Leray spectral sequence argument shows that

$$
H_{c}^{*}(\hat{X}, \mathbb{C})=H_{c}^{*}\left(G \backslash \hat{X}, q_{!} \mathbb{C}\right)
$$

The constant function with value 1 on $G$ defines an idempotent in the Hecke algebra of $G$ which induces a projector onto the $G$-invariant part on any smooth $G$-module and consequently on the $G$-equivariant sheaf $q$ ! $\mathbb{C}$. We obtain

$$
H_{G, c}^{*}(p t, X)=H_{c}^{*}\left(G \backslash \hat{X}, q_{!} \mathbb{C}\right)^{G}=H_{c}^{*}\left(G \backslash \hat{X},\left(q_{!} \mathbb{C}\right)^{G}\right)=H_{c}^{*}(G \backslash \hat{X}, \mathbb{C})
$$

Thus our construction coincides with the equivariant cohomology groups which were introduced, for a finite group $G$, in $[\mathrm{BC}] \S 1$.

B. Let $\mathcal{Y}$ be an abstract simplicial complex of finite dimension $d$ on which $G$ acts simplicially in such a way that the stabilizer $G_{\sigma}$ of each simplex $\sigma$ is a compact open subgroup. For convenience we assume that $G$ acts "type-preserving" on $\mathcal{Y}$ meaning that $G_{\sigma}$ fixes the vertices of $\sigma$ (this can always be achieved by passing to the barycentric subdivision) and we fix once and for all a $G$-invariant orientation of $\mathcal{Y}$. Assume $Y=|\mathcal{Y}|$ to be the topological realization of $\mathcal{Y}$. The $G$-action on $Y$ then is proper. Let $\mathcal{Y}_{q}$ denote the set of $q$-dimensional simplices of $\mathcal{Y}$ and, for any $\sigma \in \mathcal{Y}_{q}$, let $\sigma^{\circ} \subseteq Y$ be the corresponding "open" simplex. The preimage of $\sigma^{\circ}$ in $\hat{Y}$ is, by our simplifying assumption, equal to $G_{\sigma} \times \sigma^{\circ}$. Let $i_{\sigma}: G_{\sigma} \hookrightarrow G_{0}$ and $j_{\sigma}: G_{\sigma} \times \sigma^{\circ} \hookrightarrow \hat{Y}$ denote the inclusion maps. The filtration of $\hat{Y}$ by the preimages of the skeleta of $Y$ gives rise to the spectral sequence

$$
E_{1}^{p, q}=\underset{\sigma \in \mathcal{Y}_{p}}{\bigoplus^{p+q}} \varphi_{Y !}\left(j_{\sigma !} j_{\sigma}^{*} \mathbb{C}\right) \Longrightarrow R^{p+q} \varphi_{Y !} \mathbb{C}
$$


Since the map $\varphi_{Y}$ restricted to $G_{\sigma} \times \sigma^{\circ}$ simply is the projection onto the first factor $G_{\sigma}$ we have (by our fixing of an orientation)

$$
R^{q} \varphi_{Y !}\left(j_{\sigma !} j_{\sigma}^{*} \mathbb{C}\right)= \begin{cases}i_{\sigma !} i_{\sigma}^{*} \mathbb{C} & \text { for } q=\operatorname{dim}(\sigma) \\ 0 & \text { otherwise }\end{cases}
$$

Hence the spectral sequence degenerates. This reasoning actually shows (compare [KS] Ex. II.21) that $R \varphi_{Y !} \mathbb{C}$ is naturally quasi-isomorphic to the complex

$$
\underset{\sigma \in \mathcal{Y}_{0}}{\oplus} i_{\sigma !} i_{\sigma}^{*} \mathbb{C} \longrightarrow \cdots \longrightarrow \underset{\sigma \in \mathcal{Y}_{d}}{\oplus} i_{\sigma !} i_{\sigma}^{*} \mathbb{C}
$$

the differentials of which are induced in the obvious way by the simplicial coboundary for $\mathcal{Y}\left(\mathcal{Y}\right.$ is locally finite!). Let $G \mathcal{Y}_{q}$ denote a set of representatives for the $G$-orbits in $\mathcal{Y}_{q}$. We then have

$$
\underset{\sigma \in \mathcal{Y}_{q}}{\oplus} i_{\sigma !} i_{\sigma}^{*} \mathbb{C}=\underset{\sigma \in G \backslash \mathcal{Y}_{q}}{\oplus} c-\operatorname{Ind}_{G_{\sigma}}^{G}\left(i_{\sigma !} i_{\sigma}^{*} \mathbb{C}\right)
$$

where

$$
c \text {-Ind } G_{\sigma}^{G}: \operatorname{Sh}_{G_{\sigma}}\left(G_{0}\right) \longrightarrow \operatorname{Sh}_{G}\left(G_{0}\right)
$$

is the functor of compact induction in [Sch] $\S 3$. From [Sch] 3.2 we obtain

$$
\begin{aligned}
\operatorname{Hom}_{\mathrm{Sh}_{G}\left(G_{0}\right)}\left(\underset{\sigma \in \mathcal{Y}_{q}}{\oplus} i_{\sigma !} i_{\sigma}^{*} \mathbb{C}, \mathcal{S}\right) & =\prod_{\sigma \in G \backslash \mathcal{Y}_{q}} \operatorname{Hom}_{\mathrm{Sh}_{G_{\sigma}}\left(G_{0}\right)}\left(i_{\sigma !} i_{\sigma}^{*} \mathbb{C}, \mathcal{S}\right) \\
& =\prod_{\sigma \in G \backslash \mathcal{Y}_{q}} \mathcal{S}\left(G_{\sigma}\right)^{G_{\sigma}}
\end{aligned}
$$

for any sheaf $\mathcal{S}$ in $\operatorname{Sh}_{G}\left(G_{0}\right)$. Since the right hand side is an exact functor in $\mathcal{S}$ we see that our complex (1) consists of projective objects in $\operatorname{Sh}_{G}\left(G_{0}\right)$. It follows that $\operatorname{Ext}_{\mathrm{Sh}_{G}\left(G_{0}\right)}^{*}\left(R \varphi_{Y !} \mathbb{C}, \mathcal{S}\right)$ is equal to the homology

$$
h_{-*}\left[\prod_{\sigma \in G \backslash \mathcal{Y}_{d}} \mathcal{S}\left(G_{\sigma}\right)^{G_{\sigma}} \longrightarrow \ldots \longrightarrow \prod_{\sigma \in G \backslash \mathcal{Y}_{0}} \mathcal{S}\left(G_{\sigma}\right)^{G_{\sigma}}\right]
$$

In order to deduce from that a formula for $H_{G, c}^{*}(Y, X)$ we fix a $c$-soft resolution $\mathbb{C} \stackrel{\sim}{\longrightarrow} I \cdot$ in $\operatorname{Sh}_{G}(\hat{X})$. Put

$$
\hat{X}_{\sigma}:=\varphi_{X}^{-1}\left(G_{\sigma}\right)=\left\{(g, x) \in \hat{X}: g \in G_{\sigma}\right\} .
$$

We then have

$$
H_{G, c}^{*}(Y, X)=h_{-*}\left[\prod_{\sigma \in G \backslash \mathcal{Y} .} \Gamma_{c}\left(\hat{X}_{\sigma}, I^{-\cdot}\right)^{G_{\sigma}}\right]
$$


Note that $\mathcal{S}\left(G_{\sigma}\right)^{G_{\sigma}}=\mathcal{S}\left(G_{\sigma}\right)_{G_{\sigma}}$ because of the compactness of $G_{\sigma}$. If we assume $Y$ to be $G$-compact, i.e., the sets $G \mathcal{Y}_{q}$ to be finite we therefore may write

$$
\prod_{\sigma \in G \backslash \mathcal{Y}_{q}} \mathcal{S}\left(G_{\sigma}\right)^{G_{\sigma}}=\underset{\sigma \in G \backslash \mathcal{Y}_{q}}{\oplus} \mathcal{S}\left(G_{\sigma}\right)_{G_{\sigma}}=\left(\underset{\sigma \in \mathcal{Y}_{q}}{\oplus} \mathcal{S}\left(G_{\sigma}\right)\right)_{G} .
$$

In particular, for $G$-compact $Y$, the formula (2) becomes

$$
H_{G, c}^{*}(Y, X)=h_{-*}\left[\underset{\sigma \in \mathcal{Y} .}{\oplus} \Gamma_{c}\left(\hat{X}_{\sigma}, I^{-\cdot}\right)\right]_{G} .
$$

Now let $\mathcal{Y}$ be an arbitrary abstract simplicial complex with a simplicial and type-preserving $G$-action such that each stabilizer $G_{\sigma}$ is compact open in $G$. The topological realization $|\mathcal{Y}|$ then carries a continuous and proper $G$-action. By an easy limit argument the identity (3) generalizes to

$$
H_{G, !}^{*}(|\mathcal{Y}|, X)=h_{-*}\left[\underset{\sigma \in \mathcal{Y} .}{\oplus} \Gamma_{c}\left(\hat{X}_{\sigma}, I^{-\cdot}\right)\right]_{G} .
$$

In particular we see that, for $G$ discrete and $X=p t$, our groups $H_{G, !}^{-*}(|\mathcal{Y}|, p t)$ coincide with the cosheaf homology groups $H_{*}^{G}(|\mathcal{Y}|, \mathbb{C})$ introduced in $[\mathrm{BCH}]$ 7.2. For the following construction let us make the technical assumption that

$G_{0}$ is equal to the union of all compact open subgroups of $G$.

This is certainly satisfied in all interesting applications. Let $\mathcal{E}:=\bigcup_{H} G / H$ denote the disjoint union of all coset spaces $G / H$ for $H$ a compact open subgroup of $G$ and define $\mathcal{E}$. to be the simplicial set of finite sequences in $\mathcal{E}$. Its topological realization $\underline{E} G:=|\mathcal{E}$.$| is the space considered in [\mathrm{BC}]$ App. 3 and $[\mathrm{BCH}]$ App. 1. We may apply our above discussion to the barycentric subdivision of $\mathcal{E}$. By comparing the above formula (4) with the corresponding formula in [Sch] $\S 4$ we obtain the duality formula

$$
H_{G, !}^{*}(\underline{E} G, X)=H_{-*}^{G}(X)
$$

provided $\hat{X}$ has finite $c$-cohomological dimension. Here the groups on the right hand side are the delocalized equivariant homology groups of $X$ introduced in [Sch] $\S 1$.

C. Assume $G$ to be finite and $\mathcal{Y}$ to be a finite abstract simplicial complex with a simplicial and type-preserving $G$-action. Using the same ideas as in A. and B. one easily shows that $H_{G, c}^{*}(p t,|\mathcal{Y}|)$ coincides with the equivariant Bredon cohomology of $|\mathcal{Y}|$ (compare [Bre] I.6) for the following contravariant coefficient 
system on the orbit category: To an orbit $G / H$ corresponds the group of $\mathbb{C}$ valued class functions on $H$. For this reason our later results will contain as a special case the results of [Slo].

D. We finally come to the comparison with the equivariant cohomology groups $H^{*}(X, G)$ as defined in $[\mathrm{BC}] \S 12$. In this section we always assume that $G$ is discrete, that $X$ is a paracompact $C^{\infty}$-manifold of some fixed dimension, and that the $G$-action on $X$ is a $C^{\infty}$-action. Then $\hat{X}$ again is a paracompact $C^{\infty}$ manifold on which $G$ acts through a $C^{\infty}$-action. Let $T \hat{X}$ denote the tangent bundle over $\hat{X}$. We recall that the groups in question are defined as the relative singular homology groups

$$
H^{*}(X, G):=\bigoplus_{j \geq 0} H_{2 j+*}(E G \underset{G}{\times} T \hat{X},(E G \underset{G}{\times} T \hat{X}) \backslash\{0\} ; \mathbb{C}) ;
$$

here $E G$ denotes, as usual, a fixed model for the universal free $G$-action. We claim that there is a canonical isomorphism

$$
\underset{j \in \mathbf{Z}}{\oplus} H_{G, !}^{2 j+*}(\underline{E} G, X)=H^{*}(X, G)
$$

First of all we note that although $\hat{X}$ may have connected components of varying dimension those dimensions are bounded above by $\operatorname{dim}(X)$. It follows that $\hat{X}$ has finite $c$-cohomological dimension so that we have our duality formula at our disposal. It therefore suffices to establish a canonical isomorphism

$$
\bigoplus_{j \in \mathbf{Z}} H_{2 j-*}^{G}(X)=H^{*}(X, G)
$$

or rather, if we insert the definitions on both sides,

$$
\underset{j \in \mathbf{Z}}{\oplus} H_{2 j-*}(\hat{X}, G ; \mathbb{C})=\underset{j \geq 0}{\oplus} H_{2 j+*}(E G \underset{G}{\times} T \hat{X},(E G \underset{G}{\times} T \hat{X}) \backslash\{0\} ; \mathbb{C})
$$

If we decompose $\hat{X}$ as a disjoint union of $G$-equivariant equidimensional manifolds then both sides in the above formula decompose correspondingly. What we are after therefore is a consequence of the following more general and more precise claim. If $d$ denotes the dimension of $X$ then we have a canonical isomorphism

$$
H_{*-2 d}(X, G ; \mathbb{C})=H_{*}(E G \underset{G}{\times} T X,(E G \underset{G}{\times} T X) \backslash\{0\} ; \mathbb{C}) .
$$

By the Thom isomorphism ([Spa] p. 283, Ex. 6) applied to the vector bundle $E G \times \underset{G}{\times} T X$ over $E G \underset{G}{\times} X$ (because of our paracompactness assumption that vector bundle contains a sphere bundle and the bundle projection is a fibration) the right hand side can be expressed as the homology

$$
H_{*}(E G \underset{G}{\times} T X,(E G \underset{G}{\times} T X) \backslash\{0\} ; \mathbb{C})=H_{*-d}\left(E G \underset{G}{\times} X, \tau^{\prime}\right)
$$


of the local system $\tau^{\prime}$ on $E G \underset{G}{\times} X$ defined by

$$
(E G \underset{G}{\times} X) \ni y \longmapsto \tau_{y}^{\prime}:=H_{d}\left((E G \underset{G}{\times} T X)_{y},(E G \underset{G}{\times} T X)_{y} \backslash\{0\} ; \mathbb{C}\right) .
$$

On $X$ we have a similar local system $\tau$ defined by

$$
x \longmapsto \tau_{x}:=H_{d}\left((T X)_{x},(T X)_{x} \backslash\{0\} ; \mathbb{C}\right)
$$

which actually is $G$-equivariant. If $y$ is the $G$-orbit of the point $(e, x) \in E G \times X$ then there is the linear isomorphism

$$
\begin{array}{rl}
(T X)_{x} & \cong \\
v & \longmapsto G \times \underset{G}{\longmapsto}(E X)_{y} \\
v & G \text {-orbit of }(e, v) .
\end{array}
$$

This can be expressed more invariantly as a homeomorphism

$$
G \backslash\left[\bigcup_{(e, x) \in y}(T X)_{x}\right] \stackrel{\sim}{\longrightarrow}(E G \times \underset{G}{\times} T X)_{y}
$$

It induces a canonical isomorphism

$$
\left[\underset{(e, x) \in y}{\oplus} \tau_{x}\right]_{G} \stackrel{\cong}{\longrightarrow} \tau_{y}^{\prime}
$$

If we denote, following [Spa] p. $281 / 282$, by $\Delta(Y, \gamma)$ the singular chain complex of a local system $\gamma$ on the space $Y$ then the last isomorphism furthermore induces a natural isomorphism

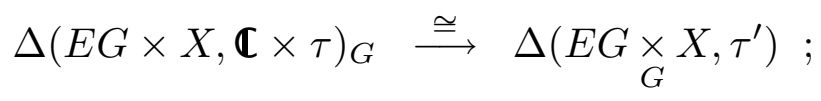

here $\mathbb{C} \times \tau$ denotes the outer tensor product of the constant local system $\mathbb{C}$ on $E G$ and the local system $\tau$ on $X$. Moreover the complex $\Delta(E G \times X, \mathbb{C} \times \tau)$ consists of free $\mathbb{C}[G]$-modules. (All this is a straightforward generalization of, e.g., [McL] IV.11.) The Künneth formula ([Spa] p. 282, Ex. 8) gives a natural chain equivalence

$$
\Delta(E G, \mathbb{C}) \otimes \Delta(X, \tau) \stackrel{\cong}{\longrightarrow} \Delta(E G \times X, \mathbb{C} \times \tau) .
$$

But the space $E G$ is contractible. It follows that $\Delta(X, \tau)$ and $\Delta(E G \times X, \mathbb{C} \times \tau)$ are naturally quasi-isomorphic as complexes of $G$-modules. At this point we have expressed the right hand side of our claim

$$
\begin{aligned}
H_{*}(E G \underset{G}{\times} T X,(E G \underset{G}{\times} T X) \backslash\{0\} ; \mathbb{C}) & =h_{*-d}\left(\Delta(E G \times X, \mathbb{C} \times \tau)_{G}\right) \\
& =H_{*-d}(G, \Delta(E G \times X, \mathbb{C} \times \tau)) \\
& =H_{*-d}(G, \Delta(X, \tau))
\end{aligned}
$$


as the group hyperhomology of the complex of $G$-modules $\Delta(X, \tau)$. Integrating a form over a singular chain leads to a $G$-equivariant quasi-isomorphism

$$
\Delta(X, \tau) \stackrel{\sim}{\longrightarrow} \mathcal{C}^{c}(X, \tau)
$$

into the complex of global $\tau$-valued currents with compact support on $X$ viewed as a homological complex (compare [Rha]).

On the other hand the left hand side of our claim is, by definition ([Sch] $\S 1$ ),

$$
H_{*-2 d}(X, G ; \mathbb{C})=H_{*-2 d}\left(G, \Gamma_{c}\left(X, I^{-\cdot}\right)\right)
$$

the group hyperhomology of the complex of global sections with compact support $\Gamma_{c}\left(X, I^{-\cdot}\right)$ where $\mathbb{C} \stackrel{\sim}{\longrightarrow} I$ is a resolution in $\operatorname{Sh}_{G}(X)$ of the constant sheaf $\mathbb{C}$ on $X$ with the property that all the sheaves $I^{j}$ are $c$-soft. By the Poincaré lemma ([KS] 2.9.7) we may take the resolution $\mathbb{C} \stackrel{\sim}{\longrightarrow} \Omega_{X}$ by the complex of germs of $C^{\infty}$-forms on $X$. Let us write $\Omega_{c}^{*}(X):=\Gamma_{c}\left(X, \Omega_{X}^{*}\right)$. To arrive at our claim it remains to exhibit a $G$-equivariant quasi-isomorphism

$$
\Omega_{c}^{d-\cdot}(X) \stackrel{\sim}{\longrightarrow} \mathcal{C}^{c}(X, \tau) .
$$

But this is Poincaré duality: The map is given by $\omega \mapsto\left[\eta \mapsto \int_{X} \eta \wedge \omega\right]$.

Let us assume in addition that the $G$-action on $X$ is proper. Then also the $G$-action on $\hat{X}$ is proper so that $G \backslash \hat{X}$ is a locally compact Hausdorff space. Combining the above discussion with $[\mathrm{BC}] 12.4$ we obtain a canonical isomorphism

$$
H_{G, !}^{*}(\underline{E} G, X)=H_{c}^{*}(G \backslash \hat{X}, \mathbb{C})
$$

E. We finally come back to the case of a compact group $G$. Let $Y$ be a $G$-space on which $G$ acts through a finite quotient, i.e., such that there is an open normal subgroup $H \subseteq G$ which acts trivially on $Y$. We then have the $H$-invariant decomposition

$$
\hat{Y}=\bigcup_{g \in G / H} g H \times Y^{g}
$$

or equivalently

$$
\varphi_{Y}^{-1}(g H)=g H \times Y^{g} \quad \text { for any } g \in G .
$$

It then is a consequence of base change that

$$
R^{q} \varphi_{Y !} \mathbb{C} \mid g H=\text { constant sheaf with value } H_{c}^{q}\left(Y^{g}, \mathbb{C}\right)
$$

as an $H$-equivariant sheaf. 
To exploit this fact we consider an arbitrary $G$-space $Z$ which is totally disconnected. Moreover, we let $\mathcal{S}$ be a $G$-equivariant sheaf on $Z$ which is uniformly locally constant. By the latter we mean that there is an open subgroup $U \subseteq G$ and a covering $Z=\dot{\bigcup}_{i} Z_{i}$ by pairwise disjoint $U$-invariant compact open subsets $Z_{i}$ such that each restriction $\mathcal{S} \mid Z_{i}$ as an $U$-equivariant sheaf is constant, i.e., is isomorphic to the inverse image of a smooth $U$-module $S_{i}$ with respect to the map $Z_{i} \rightarrow p t$. For any other $G$-equivariant sheaf $\mathcal{T}$ on $Z$ we then have

$\operatorname{Hom}_{\mathrm{Sh}_{G}(Z)}(\mathcal{S}, \mathcal{T})=\left[\prod_{i} \operatorname{Hom}_{\mathrm{Sh}_{U}\left(Z_{i}\right)}\left(\mathcal{S}\left|Z_{i}, \mathcal{T}\right| Z_{i}\right)\right]^{G}=\left[\prod_{i} \operatorname{Hom}_{\mathbb{C}}\left(S_{i}, \mathcal{T}\left(Z_{i}\right)\right)^{U}\right]^{G}$

Since on a compact and totally disconnected space any sheaf is soft the section functors $\mathcal{T} \longmapsto \mathcal{T}\left(Z_{i}\right)$ are exact. Furthermore, since the category of smooth $U$-modules is semisimple the functors $\mathcal{T} \longrightarrow \operatorname{Hom}_{\mathbb{C}}\left(S_{i}, \mathcal{T}\left(Z_{i}\right)\right)^{U}$ still are exact. Passing to the $G$-invariants on the right hand side actually amounts to passing to the invariants for an appropriate finite quotient of $G$ which is exact again. Altogether it follows that the functor $\operatorname{Hom}_{\mathrm{Sh}_{G}(Z)}(\mathcal{S},$.$) is exact on \mathrm{Sh}_{G}(Z)$. Let now $\mathcal{S}$ be an arbitrary (possibly unbounded in both directions) complex of sheaves in $\operatorname{Sh}_{G}(Z)$ all whose cohomology sheaves $h^{*}(\mathcal{S})$ are uniformly locally constant. By the above exactness result the identity map on $h^{*}(\mathcal{S})$ lifts to a map $h^{*}\left(\mathcal{S}^{*}\right) \longrightarrow \operatorname{ker}\left(\mathcal{S}^{*} \stackrel{d}{\rightarrow} \mathcal{S}^{*+1}\right) \subseteq \mathcal{S}^{*}$. These maps constitute a quasi-isomorphism of complexes $h^{\cdot}\left(\mathcal{S}^{*}\right) \longrightarrow \mathcal{S}^{*}$ in $\operatorname{Sh}_{G}(Z)$ where $h^{\prime}\left(\mathcal{S}^{*}\right)$ is considered as a complex with zero differentials. Let $\mathcal{T}$ be be any other complex in $\operatorname{Sh}_{G}(Z)$ bounded below and let $\mathcal{T} \cdot \stackrel{\sim}{\longrightarrow} I$ be an injective resolution. We obtain

$$
\begin{aligned}
& \operatorname{Ext}_{\mathrm{Sh}_{G}(Z)}^{*}\left(\mathcal{S}, \mathcal{T}^{\cdot}\right)=\operatorname{Ext}_{\mathrm{Sh}_{G}(Z)}^{*}(h \cdot(\mathcal{S} \cdot), \mathcal{T} \cdot)=h^{*}\left(\operatorname{Hom}_{\mathrm{Sh}_{G}(Z)}\left(h^{\cdot}\left(\mathcal{S}^{\cdot}\right), I^{\cdot}\right)\right) \\
& =\prod_{q} h^{q+*}\left(\operatorname{Hom}_{\mathrm{Sh}_{G}(Z)}\left(h^{q}\left(\mathcal{S}^{\cdot}\right), I^{\cdot}\right)\right)=\prod_{q} \operatorname{Hom}_{\mathrm{Sh}_{G}(Z)}\left(h^{q}\left(\mathcal{S}^{\cdot}\right), h^{q+*}\left(I^{\cdot}\right)\right) \\
& =\prod_{q} \operatorname{Hom}_{\mathrm{Sh}_{G}(Z)}\left(h^{q}\left(\mathcal{S}^{\cdot}\right), h^{q+*}\left(\mathcal{T}^{\cdot}\right)\right)
\end{aligned}
$$

where the fourth identity again comes from the above exactness result.

In our initial situation this specializes to the following result. If $G$ is compact, $X$ is an arbitrary $G$-space, and $Y$ is a $G$-space on which $G$ acts through a finite quotient then we have

$$
\begin{aligned}
H_{G, c}^{*}(Y, X) & =\prod_{q} \operatorname{Hom}_{\mathrm{Sh}_{G}(Z)}\left(R^{q} \varphi_{Y !} \mathbb{C}, R^{q+*} \varphi_{X !} \mathbb{C}\right) \\
& =\prod_{q} \operatorname{Hom}_{\operatorname{Mod}(\Gamma(G, \mathbb{C}), G)}\left(H_{c}^{q}(\hat{Y}, \mathbb{C}), H_{c}^{q+*}(\hat{X}, \mathbb{C})\right) .
\end{aligned}
$$

Because of this formula the bivariant Chern character which we will construct in section 3 generalizes the non-equivariant construction in [Mas]. 


\section{The cohomological Chern character for a profinite group $G$}

In this section the group $G$ always is compact. Initially we also assume the $G$ space $X$ to be compact. Given a $G$-equivariant vector bundle $E \longrightarrow X$ (compare [Seg] $\S 1)$ its pullback $\pi_{X}^{*} E \longrightarrow \hat{X}$ is a $G$-equivariant vector bundle on $\hat{X}$. A point in $\pi_{X}^{*} E$ is a triple $(e, g, x)$ where $e \in E_{x}, g \in G$, and $x \in X$ such that $g x=x$. It is straightforward to check that

$$
\begin{array}{cccc}
\alpha: & \pi_{X}^{*} E & \longrightarrow & \pi_{X}^{*} E \\
(e, g, x) & \longmapsto & (g e, g, x)
\end{array}
$$

is an automorphism of $G$-equivariant vector bundles. We claim that $\alpha$ has finite order. In order to see this we may assume, by [Seg] 2.4, that $E$ is of the form $E=X \times V$ where $V$ is a finite dimensional $\mathbb{C}$-vector space with a continuous $G$-action and where the $G$-action on $E$ is diagonal. Since the only compact and totally disconnected subgroups of the Lie group $G L(V)$ are the finite ones it follows that an appropriate open normal subgroup $H \subseteq G$ acts trivially on $V$. We then clearly have $\alpha^{[G: H]}=i d$. This allows us to view $\pi_{X}^{*} E$ as a vector bundle on $\hat{X}$ which is equivariant for the profinite completion $\hat{\mathbb{Z}}$ of the infinite cyclic group $\mathbb{Z}$; of course we let act $\hat{\mathbb{Z}}$ trivially on the space $\hat{X}$. Moreover $\pi_{X}^{*} E$ being also $G$-equivariant its class in $K_{\hat{\mathbf{z}}}^{0}(\hat{X})$ is fixed by the natural action of $G$ on that group. Altogether we have constructed a natural map between equivariant $K$-groups

$$
\begin{aligned}
K_{G}^{0}(X) & \longrightarrow K_{\hat{\mathbf{z}}}^{0}(\hat{X})^{G} \\
{[E] } & \longmapsto\left[\pi_{X}^{*} E\right] .
\end{aligned}
$$

According to [Seg] 2.2 one has

$$
K_{\hat{\mathbf{Z}}}^{0}(\hat{X})=K^{0}(\hat{X}) \underset{\mathbf{Z}}{\otimes} R(\hat{\mathbb{Z}})
$$

where $R(\hat{\mathbb{Z}})$ denotes the representation ring of the group $\hat{\mathbb{Z}}$. Taking the trace of the element $1 \in \mathbb{Z}$ on a representation of $\hat{\mathbb{Z}}$ defines a ring homomorphism

$$
\tau: R(\hat{\mathbb{Z}}) \longrightarrow \mathbb{C}
$$

Let

$$
\Pi: K_{G}^{0}(X) \longrightarrow\left(K_{\hat{\mathbf{Z}}}^{0}(\hat{X})_{\mathbb{C}}\right)^{G}=\left(\left(K^{0}(\hat{X}) \underset{\mathbf{Z}}{\otimes} R(\hat{\mathbb{Z}})\right)_{\mathbb{C}}\right)^{G} \stackrel{i d \otimes \tau}{\longrightarrow}\left(K^{0}(\hat{X})_{\mathbb{C}}\right)^{G}
$$

denote the composed map; the subscript $\mathbb{C}$ stands for tensoring by $\mathbb{C}$.

Using the ordinary Chern character for $\hat{X}$ we define the equivariant Chern character

$$
\operatorname{ch}: K_{G}^{0}(X) \longrightarrow \underset{j \geq 0}{\oplus} H_{G, c}^{2 j}(p t, X)
$$


by the commutativity of the following diagram

$$
\begin{array}{cccc}
K_{G}^{0}(X) & \stackrel{\mathrm{ch}}{\longrightarrow} & \underset{j \geq 0}{\oplus} & H_{G, c}^{2 j}(p t, X) \\
\Pi \downarrow & & \downarrow= \\
\left(K^{0}(\hat{X})_{\mathbb{C}}\right)^{G} & \stackrel{\mathrm{ch}}{\longrightarrow} & \underset{j \geq 0}{\oplus} & H^{2 j}(\hat{X}, \mathbb{C})^{G} .
\end{array}
$$

We now consider the case of a locally compact $G$-space $X$ which is not compact. Let $X^{+}=X \cup\{+\}$ denote the one-point compactification of $X$; it is a $G$-space if we let $G$ act trivially on the point at infinity. Recall ([Seg] $\S 2$ ) that the equivariant $K^{0}$ of $X$ is defined as

$$
K_{G}^{0}(X):=\operatorname{ker}\left(K_{G}^{0}\left(X^{+}\right) \longrightarrow K_{G}^{0}(\{+\})\right)
$$

On the other hand using [Seg] 2.9, resp. the relative cohomology sequence for cohomology with compact support, we have

$$
K_{\hat{\mathbf{Z}}}^{0}(\hat{X})=\operatorname{ker}\left(K_{\hat{\mathbf{Z}}}^{0}\left(\left(X^{+}\right)^{\wedge}\right) \longrightarrow K_{\hat{\mathbf{Z}}}^{0}\left(\{+\}^{\wedge}\right)\right)
$$

resp.

$$
H_{G, c}^{*}(p t, X)=\operatorname{ker}\left(H_{G, c}^{*}\left(p t, X^{+}\right) \longrightarrow H_{G, c}^{*}(p t,\{+\})\right) .
$$

Hence the above maps $\Pi$ and ch for $X^{+}$induce corresponding maps for $X$.

\section{Proposition 1:}

The $\operatorname{map} \Pi: K_{G}^{0}(X)_{\mathbb{C}} \stackrel{\cong}{\longrightarrow}\left(K^{0}(\hat{X})_{\mathbb{C}}\right)^{G}$ is an isomorphism.

Proof: The $G$-space $X$ is the projective limit of the $G$-spaces $H \backslash X$ where $H$ runs through the open normal subgroups of $G$. Correspondingly $\hat{X}$ is the projective limit of the $(H \backslash X)^{\wedge}$. The continuity property of $K_{G}^{0}$ ([Seg] Remark after 2.11) then gives

$$
K_{G}^{0}(X)=\lim _{\vec{H}} K_{G}^{0}(H \backslash X) \quad \text { and } \quad K^{0}(\hat{X})=\underset{\vec{H}}{\lim } K^{0}\left((H \backslash X)^{\wedge}\right)
$$

Moreover by applying this reasoning directly to $\hat{X}$ we see that $K^{0}(\hat{X})_{\mathbb{C}}$ is a smooth $G$-module. Hence

$$
\left(K^{0}(\hat{X})_{\mathbb{C}}^{G}=\underset{H}{\lim }\left(K^{0}\left((H \backslash X)^{\wedge}\right) \mathbb{C}\right)^{G}\right.
$$


Altogether this shows that it suffices to prove the assertion under the additional assumption that $G$ acts on $X$ through a finite quotient. This we will establish by induction with respect to the index of the subgroup

$$
G_{X}:=\{g \in G: g \text { fixes } X \text { pointwise }\}
$$

in $G$. If $G_{X}=G$, i.e., if $G$ acts trivially on $X$ we have

$$
K_{G}^{0}(X)_{\mathbb{C}}=R(G)_{\mathbb{C}} \underset{\mathbb{C}}{\otimes} K^{0}(X)_{\mathbb{C}}
$$

and

$$
\begin{aligned}
\left(K^{0}(\hat{X})_{\mathbb{C}}\right)^{G} & =\left(K^{0}(G \times X)_{\mathbb{C}}\right)^{G}=\left(K^{0}(G)_{\mathbb{C}} \underset{\mathbb{C}}{\otimes} K^{0}(X)_{\mathbb{C}}\right)^{G} \\
& =\left(K^{0}(G)_{\mathbb{C}}\right)^{G} \underset{\mathbb{C}}{\otimes} K^{0}(X)_{\mathbb{C}} .
\end{aligned}
$$

The map $\Pi$ is the identity on the second factor and on the first factor is the map which associates to an irreducible $G$-representation its character. Hence $\Pi$ is an isomorphism.

Let now $X$ be an arbitrary locally compact $G$-space for which $G_{X}$ is of finite index in $G$. We fix a set $\mathcal{P}$ of representatives for the conjugacy classes of subgroups of $G$ which contain $G_{X}$. This set $\mathcal{P}$ is finite and is partially ordered by

$$
H^{\prime} \leq H \text { if } H^{\prime} \subseteq g H g^{-1} \text { for some } g \in G .
$$

For $H \in \mathcal{P}$ we put

$$
X(H):=\{x \in X: H \text { is the stabilizer of } x\} \text { and } X[H]:=G \cdot X(H) .
$$

Since the stabilizer of a point increases under specialization these subsets are locally closed in $X$. More precisely we have

$$
\text { closure of } X[H] \subseteq \bigcup_{H^{\prime} \leq H} X\left[H^{\prime}\right]
$$

Applying excision ([Seg] 2.9) to this finite stratification of $X$ we are reduced to the case that $X=X[H]$ for some $G_{X} \subseteq H \subseteq G$. The map

$$
\begin{aligned}
G \times X(H) & \longrightarrow X[H] \\
(g, x) & \longmapsto g x
\end{aligned}
$$

induces a homeomorphism

$$
G \underset{N(H)}{\times} X(H) \stackrel{\sim}{\longrightarrow} X[H]
$$


showing that $X[H]$ is "induced" from the $N(H)$-space $X(H)$; here $N(H)$ denotes the normalizer of $H$ in $G$. Similarly

$$
\begin{aligned}
G \underset{N(H)}{\times} X(H)^{\wedge} & \stackrel{\sim}{\longrightarrow} X[H]^{\wedge} \\
(g,(h, x)) & \longmapsto\left(g h g^{-1}, g x\right)
\end{aligned}
$$

is a homeomorphism. Using the behaviour of $K_{G}^{0}$ with respect to "induction" ([Seg] $\S 2$ ex. (iii)) we obtain

$$
K_{G}^{0}(X[H])_{\mathbb{C}}=K_{N(H)}^{0}(X(H))_{\mathbb{C}} \text { and }\left(K^{0}\left(X[H]^{\wedge}\right)_{\mathbb{C}}\right)^{G}=\left(K^{0}\left(X(H)^{\wedge}\right)_{\mathbb{C}}\right)^{N(H)}
$$

which further reduces us to the $N(H)$-space $X(H)$. If $H$ is strictly bigger than $G_{X}$ we are done by the induction hypothesis. It therefore remains to settle the case where $G / G_{X}$ is a finite group which acts freely on $X$. In this situation $X$ has an open $G$-invariant covering by subsets of the form $G_{G_{X}} Y$ where $G_{X}$ acts trivially on $Y$. Our assertion holds for those subsets by the same argument as above, then for finite unions of them by a Mayer-Vietoris argument ([Seg] 2.9), and finally for $X$ itself by another continuity argument ([Seg] 2.12).

\section{Corollary 2:}

The equivariant Chern character

$$
\operatorname{ch}: K_{G}^{0}(X)_{\mathbb{C}} \cong \underset{j \geq 0}{\bigoplus} H_{G, c}^{2 j}(p t, X)
$$

is an isomorphism.

Proof: This follows from the Proposition since the ordinary Chern character for the space $\hat{X}$ is known to be rationally an isomorphism ([Kar] V.3.25).

The higher equivariant $K$-groups of a locally compact $G$-space $X$ are defined ([Seg] $§ 2)$ by

$$
K_{G}^{-q}(X):=K_{G}^{0}\left(X \times \mathbb{R}^{q}\right) \text { for } q \geq 0
$$

where, of course, the $G$-action on the factor $\mathbb{R}^{q}$ is trivial. Because of $\left(X \times \mathbb{R}^{q}\right)^{\wedge}=$ $\hat{X} \times \mathbb{R}^{q}$ we have $K_{\hat{\mathbf{z}}}^{-q}(\hat{X})=K_{\hat{\mathbf{Z}}}^{0}\left(\left(X \times \mathbb{R}^{q}\right)^{\wedge}\right)$ and $H_{G, c}^{*}\left(p t, X \times \mathbb{R}^{q}\right)=H_{G, c}^{*-q}(p t, X)$. We therefore obtain the following general fact.

\section{Proposition 3:}

For any locally compact $G$-space $X$ we have an equivariant Chern character isomorphism

$$
\operatorname{ch}: K_{G}^{*}(X)_{\mathbb{C}} \stackrel{\cong}{\longrightarrow}\left(K^{*}(\hat{X})_{\mathbb{C}}\right)^{G} \stackrel{\cong}{\longrightarrow} \underset{j \in \mathbb{Z}}{\bigoplus} H_{G, c}^{*+2 j}(p t, X)
$$




\section{The bivariant Chern character for a profinite group $G$}

We want to extend our cohomological Chern character of the previous section to the bivariant setting of equivariant Kasparov $K K$-theory $K K_{G}(Y, X)$. This will be achieved in the following context. The group $G$ always is compact. For technical reasons originating in $K K$-theory we assume $G$ to be second countable and the $G$-space $X$ to be countable at infinity. On the other hand the $G$-space $Y$ will be the topological realization $Y=|\mathcal{Y}|$ of a finite simplicial complex $\mathcal{Y}$ on which $G$ is supposed to act simplicially and type-preserving through a finite quotient group.

Apart from an extension of the methods of the last section the following localization result will play a fundamental role in our construction.

\section{Proposition 1:}

Let $h \in G$ be a central element and let $\mathfrak{p} \subseteq R(G)$ denote the prime ideal in the representation ring $R(G)$ of $G$ which consists of all classes of representations with vanishing trace in $h$; the natural map

$$
K K_{G}^{*}\left(Y^{h}, X\right) \longrightarrow K K_{G}^{*}(Y, X)
$$

is an isomorphism after localization in $\mathfrak{p}$.

Proof: We actually will prove this result for an arbitrary $\sigma$-unital $G$ - $C^{*}$-algebra $B$ (instead of $X$ ) in the second variable. By filtering $Y$ by its skeleta and using the six term exact sequence in the first variable for $K K_{G}$-theory ([Ka1]§5 Thm. 5 and Thm. 7, §7 Thm. 3) we are reduced to establish a corresponding assertion for the strata of $Y$. Such a stratum is a finite disjoint union of $G$-invariant subsets of the form $G / U \times \Delta$ where $U \subseteq G$ is some open subgroup and $\Delta$ is an open standard simplex; moreover, we have $(G / U \times \Delta)^{h}=(G / U)^{h} \times \Delta$. Using $[\mathrm{Ka} 1] \S 4$ Cor. 1.2 it therefore suffices to show that

$$
K K_{G}^{*}\left((G / U)^{h} \times \Delta, B\right)_{\mathfrak{p}} \stackrel{\cong}{\longrightarrow} K K_{G}^{*}((G / U) \times \Delta, B)_{\mathfrak{p}}
$$

is an isomorphism. By Bott periodicity $([\mathrm{Ka} 1] \S 5$ Thm. 7) this is the same as showing that

$$
K K_{G}^{*}\left((G / U)^{h}, B\right)_{\mathfrak{p}} \stackrel{\cong}{\longrightarrow} K K_{G}^{*}(G / U, B)_{\mathfrak{p}}
$$

is an isomorphism. If $h \in U$ then $(G / U)^{h}=G / U$ and nothing is to prove. Let us therefore assume, for the rest of the proof, that $h \notin U$. Then $(G / U)^{h}=\emptyset$ and our claim amounts to the vanishing assertion

$$
K K_{G}^{*}(C(G / U), B)_{\mathfrak{p}}=0
$$


Letting $C(G / U)$ denote the $C^{*}$-algebra of all functions on the finite set $G / U$ we revert to the more traditional notation of using the algebra in both variables. Since $B$ is arbitrary we in fact only have to establish that

$$
K K_{G}^{0}(C(G / U), B)_{\mathfrak{p}}=0
$$

Consider for this the composed map

$$
K K_{G}^{0}(C(G / U), B) \longrightarrow K K_{U}^{0}(C(G / U), B) \longrightarrow K K_{U}^{0}(\mathbb{C}, B)
$$

where the left arrow is restriction of the group and the right arrow comes by contravariant functoriality from the homomorphism of algebras

$$
\begin{aligned}
& \mathbb{C} \longrightarrow C(G / U) \\
& a \longmapsto e_{a}(g U):= \begin{cases}a & \text { if } g \in U, \\
0 & \text { otherwise }\end{cases}
\end{aligned}
$$

By the functoriality of the Kasparov product this map $(*)$ as a map between modules for the representation rings $R(G)$ and $R(U)$, respectively, is compatible with the restriction map $R(G) \longrightarrow R(U)$. We claim that the composed map (*) is injective. Let $(\mathcal{E}, F)$ be a Kasparov $G$-module for $(C(G / U), B)$. Its image in $K K_{U}^{0}(\mathbb{C}, B)$ is the class of the Kasparov $U$-module $\left(e_{1} \mathcal{E}, e_{1} F \mid e_{1} \mathcal{E}\right)$ for $(\mathbb{C}, B)$ (compare the proof of [Ka2] Thm. 2.9.2). To see that the latter determines the class of $(\mathcal{E}, F)$ we first note that by possibly changing $(\mathcal{E}, F)$ within its class we may assume:

- $\mathcal{E}$ is a unital $C(G / U)$-module ([Ka2] Lemma 2.8).

- $F$ commutes with the $C(G / U)$-action. This can be seen as follows: The idempotents $\left(\left(g\left(e_{1}\right)\right)_{g \in G / U}\right.$ in $C(G / U)$ decompose $\mathcal{E}$. Express $F$ as an operator valued matrix in this decomposition. The requirement that $F$ commutes with the $C(G / U)$-action at least up to compact operators then implies that the offdiagonal entries of this matrix are compact operators. Hence we may modify $F$ by a compact operator to make it truly commute with the $C(G / U)$-action.

- $F$ is $G$-equivariant (by averaging over the compact group $G$ if necessary).

Under these additional assumptions it is straightforward to see that $(\mathcal{E}, F)$ can be reconstructed by inducing $\left(e_{1} \mathcal{E}, F \mid e_{1} \mathcal{E}\right)$ from $U$ to $G$, and similarly for homotopies. For purposes of later proofs we emphasize that this argument in fact shows that the map $(*)$ is bijective.

Let now $\mathfrak{n}$ denote the kernel of the restriction map $R(G) \longrightarrow R(U)$. The injectivity of $(*)$ implies that $\mathfrak{n}$ annihilates $K K_{G}^{0}(C(G / U), B)$. For the vanishing of the localization $K K_{G}^{0}(C(G / U), B)_{\mathfrak{p}}$ it is therefore sufficient to verify that

$$
\mathfrak{n} \nsubseteq \mathfrak{p}
$$


This can be done after replacing $G$ and $U$ by the finite groups $G / H$ and $U / H$, respectively, for some open normal subgroup $H \subseteq G$ contained in $U$. Since $h H$ is central in $G / H$ and is not contained in $U / H$ it then is a consequence of [Se2] Prop. 3.7(iv) and [Seg] first paragraph on p.144 (the "support" of $\mathfrak{p}$ is the cyclic subgroup $<h H>$ which is not conjugate to any subgroup of $U / H)$.

The stratification argument which appeared in the above proof is a fundamental tool which we will use again in all of the subsequent proofs (without repeating each time the details). We need two more properties of $K K_{G^{-}}$-theory. The following result holds in greater generality but, for simplicity, we indicate the argument only in the setting in which we need it.

\section{Proposition 2:}

Let another second countable profinite group $\Gamma$ act trivially on $Y$ and $X$; the Kasparov product map

$$
R(\Gamma) \underset{\mathbf{Z}}{\otimes} K K_{G}^{*}(Y, X) \stackrel{\cong}{\longrightarrow} K K_{\Gamma \times G}^{*}(Y, X)
$$

is an isomorphism.

Proof: By exactly the same stratification technique as in the proof of Prop. 1 the assertion reduces to the special case

$$
R(\Gamma) \underset{\mathbf{Z}}{\otimes} K_{U}^{*}(X) \stackrel{\cong}{\longrightarrow} K_{\Gamma \times U}^{*}(X)
$$

The usual standard argument moreover reduces us to the case where $X$ is compact and where $*=0$. This then is proved by an immediate generalization of the argument in [Seg] Prop. 2.2.

\section{Proposition 3:}

If $X$ is compact then the natural map

$$
\underset{H}{\lim _{H}} K K_{G / H}^{*}(Y, H \backslash X) \stackrel{\cong}{\longrightarrow} K K_{G}^{*}(Y, X)
$$

where $H$ runs over all open normal subgroups in $G$ which act trivially on $Y$ is an isomorphism.

Proof: By the stratification technique this reduces to the continuity property of equivariant $K$-theory in [Seg] Remark after 2.11. Formally loc. cit. establishes the isomorphism

$$
\lim _{H} K_{G}^{0}(H \backslash X) \stackrel{\cong}{\longrightarrow} K_{G}^{0}(X)
$$


But the decomposition argument in the proof of [Seg] 2.2 shows that for any $G$-equivariant vector bundle $E$ on $H \backslash X$ there is a smaller open normal subgroup $H^{\prime} \subseteq H$ in $G$ which acts trivially on $E$. Hence in the left hand limit term we my replace $G$ by $G / H$.

We finally need the following construction. Let $Y^{\prime}$ be any second countable $G$-space. By functoriality the group $G$ acts on the non-equivariant groups $K K^{*}\left(Y^{\prime}, X\right)$. It is straightforward from the definitions that the forgetful map induces a map

$$
K K_{G}^{*}\left(Y^{\prime}, X\right) \longrightarrow K K^{*}\left(Y^{\prime}, X\right)^{G} .
$$

The Kasparov product, furthermore, induces a map

$$
K K^{*}\left(Y^{\prime}, X\right) \longrightarrow \operatorname{Hom}\left(K^{*}\left(Y^{\prime}\right), K^{*}(X)\right)
$$

which by functoriality is $G$-equivariant. Together we therefore have the natural map

$$
\gamma: K K_{G}^{*}\left(Y^{\prime}, X\right) \longrightarrow \operatorname{Hom}_{G}\left(K^{*}\left(Y^{\prime}\right), K^{*}(X)\right)
$$

The main result in this section is the following "universal coefficient formula".

\section{Proposition 4:}

There is a natural isomorphism

$$
K K_{G}^{*}(Y, X) \mathbb{C} \stackrel{\cong}{\longrightarrow} \operatorname{Hom}_{\operatorname{Mod}(\Gamma(G, \mathbb{C}), G)}\left(K^{*}(\hat{Y})_{\mathbb{C}}, K^{*}(\hat{X})_{\mathbb{C}}\right)
$$

Proof: We first assume $G$ to be finite. For the moment being let $h \in G$ be a fixed element, denote its centralizer by $Z(h)$, and let $\mathfrak{p}(h)$ be the prime ideal in $R(Z(h))$ of all representations with vanishing trace in $h$. In the following chain of maps

$$
K K_{G}^{*}(Y, X) \rightarrow K K_{Z(h)}^{*}\left(Y, X^{h}\right) \rightarrow K K_{Z(h)}^{*}\left(Y, X^{h}\right)_{\mathfrak{p}(h)} \stackrel{\cong}{\longrightarrow} K K_{Z(h)}^{*}\left(Y^{h}, X^{h}\right)_{\mathfrak{p}(h)}
$$

the first comes by functoriality, the second is the natural map into the localization, and the third is the inverse of the isomorphism in Prop. 1. We will combine this one with a second chain

$$
\begin{aligned}
K K_{Z(h)}^{*}\left(Y^{h}, X^{h}\right) \rightarrow K K_{\hat{\mathbf{Z}} \times Z(h)}^{*}\left(Y^{h}, X^{h}\right)= & R(\hat{\mathbb{Z}}) \underset{\mathbf{Z}}{\otimes} K K_{Z(h)}^{*}\left(Y^{h}, X^{h}\right) \\
\downarrow & \operatorname{tr}_{1} \otimes \gamma \\
& \underset{\mathbf{Z}}{\mathbb{C}} \operatorname{Hom}_{Z(h)}\left(K^{*}\left(Y^{h}\right), K^{*}\left(X^{h}\right)\right) .
\end{aligned}
$$


Here the first map comes by functoriality from the homomorphism of groups

$$
\begin{aligned}
& \hat{\mathbb{Z}} \times Z(h) \quad \longrightarrow \quad Z(h) \\
& (n, g) \longmapsto h^{n} g \text {, }
\end{aligned}
$$

the second identity is the isomorphism in Prop. 2 (we, of course, let $\hat{\mathbb{Z}}$ act trivially on $Y^{h}$ and $X^{h}$ ), and the third map is the tensor product of taking the trace in $1 \in \hat{\mathbb{Z}}$ and the map $\gamma$ explained after Prop. 3. As far as the action of the corresponding representation rings is concerned this chain is compatible with the analogous chain of homomorphisms of rings

$$
R(Z(h)) \longrightarrow R(\hat{\mathbb{Z}} \times Z(h))=R(\hat{\mathbb{Z}}) \underset{\mathbf{Z}}{\otimes} R(Z(h)) \stackrel{\operatorname{tr}_{1} \otimes \operatorname{tr}_{e}}{\longrightarrow} \underset{\mathbb{Z}}{\mathbb{Z}} \mathbb{Z}=\mathbb{C}
$$

(the first map comes from the above homomorphism of groups, and the last map is the tensor product of taking the traces in $1 \in \hat{\mathbb{Z}}$ and in the unit element $e \in G$, respectively). We immediately notice that the composite of this latter chain is the ring homomorphism

$$
\operatorname{tr}_{h}: R(Z(h)) \longrightarrow \mathbb{C}
$$

of taking the trace in $h$. Hence the composite of the previous chain extends to a map on the localization

$$
K K_{Z(h)}^{*}\left(Y^{h}, X^{h}\right)_{\mathfrak{p}(h)} \longrightarrow \operatorname{Hom}_{Z(h)}\left(K^{*}\left(Y^{h}\right), K^{*}\left(X^{h}\right)\right) \underset{\mathbf{Z}}{\otimes} \mathbb{C}
$$

Since the $K$-theory of the finite simplicial complex $Y^{h}$ is finitely generated the Hom-functor in the right hand term commutes with the complexification. Combining this with our first chain above we obtain a natural map

$$
\gamma_{h}: K K_{G}^{*}(Y, X)_{\mathbb{C}} \longrightarrow \operatorname{Hom}_{Z(h)}\left(K^{*}\left(Y^{h}\right) \mathbb{a}, K^{*}\left(X^{h}\right) \mathbb{C}\right) .
$$

By naturality we have

$$
\gamma_{g h g^{-1}}=g \circ \gamma_{h} \circ g^{-1} \quad \text { for any } g \in G \text {. }
$$

Hence the maps $\gamma_{h}$ for $h$ varying in one conjugacy class $O$ of $G$ combine into a map

$$
\gamma_{O}: K K_{G}^{*}(Y, X)_{\mathbb{C}} \longrightarrow \operatorname{Hom}_{\operatorname{Mod}(\Gamma(O, \mathbb{C}), G)}\left(K^{*}\left(\rho_{Y}^{-1}(O)\right)_{\mathbb{C}}, K^{*}\left(\rho_{X}^{-1}(O)\right)_{\mathbb{C}}\right) .
$$

Finally, these maps $\gamma_{O}$ for $O$ running over the finitely many conjugacy classes of $G$ together form the natural map

$$
K K_{G}^{*}(Y, X)_{\mathbb{C}} \longrightarrow \operatorname{Hom}_{\operatorname{Mod}(\Gamma(G, \mathbb{C}), G)}\left(K^{*}(\hat{Y})_{\mathfrak{C}}, K^{*}(\hat{X})_{\mathbb{C}}\right)
$$


we are looking for (if $G$ is finite). That this map indeed is an isomorphism is easily derived, using the stratification technique, from Prop. 2.1.

Now let $G$ be arbitrary (profinite) but assume $X$ to be compact. Fix an open normal subgroup $N \subseteq G$ which acts trivially on $Y$. From the finite group case we obtain, for any open normal subgroup $H \subseteq G$ which is contained in $N$, the isomorphism

$$
K K_{G / H}^{*}(Y, H \backslash X)_{\mathbb{C}} \stackrel{\cong}{\longrightarrow} \operatorname{Hom}_{\operatorname{Mod}(\Gamma(G / H, \mathbb{C}), G / H)}\left(K^{*}\left(\hat{Y}^{G / H}\right)_{\mathbb{C}}, K^{*}\left((H \backslash X)^{\wedge G / H}\right) \mathbb{C}\right)
$$

where the superscript " $G / H$ " on the right hand side indicates that the space $(.)^{\wedge}$ is formed with respect to the action of $G / H$. The right hand term is equal to

$$
\begin{aligned}
& {\left[\underset{g \in G / N}{\oplus} \operatorname{Hom}_{\Gamma(g N / H, \mathbb{C})}\left(K^{*}\left((g N / H) \times Y^{g}\right)_{\mathbb{C}}, K^{*}\left((H \backslash X)^{\wedge} G / H\right) \mathbb{C}\right)\right]^{G / H}} \\
& =\left[\underset{g \in G / N}{\oplus} \operatorname{Hom}_{\mathbb{C}}\left(K^{*}\left(Y^{g}\right)_{\mathbb{C}}, K^{*}\left((H \backslash X)^{\wedge g N / H}\right) \mathbb{C}\right)\right]^{G / H}
\end{aligned}
$$

where $(H \backslash X)^{\wedge} g N / H$ is the preimage under the projection map of $g N / H$ in $(H \backslash X)^{\wedge} G / H$. We now pass to the direct limit with respect to $H$. On the left hand side of the map we obtain $K K_{G}^{*}(Y, X)_{\mathbb{C}}$ by Prop. 3. Since the $K$ theory of the finite simplicial complexes $Y^{g}$ is finitely generated we may, on the right hand side, move the direct limit into the Hom-functor. But because of

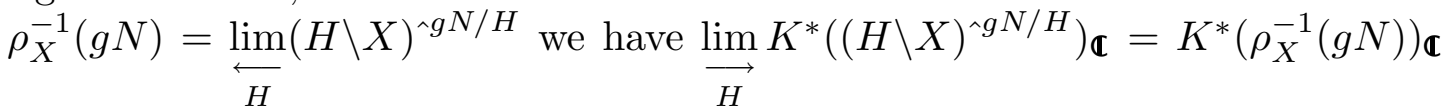
by the continuity property of $K$-theory. Hence we obtain an isomorphism

$$
K K_{G}^{*}(Y, X) \stackrel{\mathbb{C}}{\cong}\left[\underset{g \in G / N}{\bigoplus} \operatorname{Hom}_{\mathbb{C}}\left(K^{*}\left(Y^{g}\right) \mathbb{C}, K^{*}\left(\rho_{X}^{-1}(g N)\right)_{\mathbb{C}}\right)\right]^{G} .
$$

Using the Künneth theorem in $K$-theory ([Atiy] 2.7.15) we compute the target as

$$
\begin{aligned}
& {\left[\underset{g \in G / N}{\oplus} \operatorname{Hom}_{\mathbb{C}}\left(K^{*}\left(Y^{g}\right)_{\mathbb{C}}, K^{*}\left(\rho_{X}^{-1}(g N)\right)_{\mathbb{C}}\right)\right]^{G}} \\
& =\left[\underset{g \in G / N}{\oplus} \operatorname{Hom}_{\Gamma(g N, \mathbb{C})}\left(\Gamma(g N, \mathbb{C}) \underset{\mathbb{C}}{\otimes} K^{*}\left(Y^{g}\right)_{\mathbb{C}}, K^{*}\left(\rho_{X}^{-1}(g N)\right) \mathbb{C}\right)\right]^{G} \\
& =\left[\underset{g \in G / N}{\bigoplus} \operatorname{Hom}_{\Gamma(g N, \mathbb{C})}\left(\left(K^{0}(g N) \underset{\mathbf{Z}}{\otimes} K^{*}\left(Y^{g}\right)\right)_{\mathbb{d}}, K^{*}\left(\rho_{X}^{-1}(g N)\right) \mathbb{C}\right)\right]^{G} \\
& =\left[\underset{g \in G / N}{\oplus} \operatorname{Hom}_{\Gamma(g N, \mathbb{C})}\left(K^{*}\left(\rho_{Y}^{-1}(g N)\right)_{\mathbb{C}}, K^{*}\left(\rho_{X}^{-1}(g N)\right) \mathbb{\mathbb { C }}\right)\right]^{G} \\
& =\left[\operatorname{Hom}_{\Gamma(G, \mathbb{C})}\left(K^{*}(\hat{Y})_{\mathbb{C}}, K^{*}(\hat{X})_{\mathbb{C}}\right)\right]^{G} \\
& =\operatorname{Hom}_{\operatorname{Mod}(\Gamma(G, \mathbb{C}), G)}\left(K^{*}(\hat{Y})_{\mathbb{C}}, K^{*}(\hat{X})_{\mathfrak{C}}\right)
\end{aligned}
$$

(For the sake of clarity we point out that, since $G$ is second countable, the group $K^{0}(g N)$ of integer valued locally constant functions on $g N$ is free abelian.) 
Finally, the general case of a locally compact $X$ follows from the compact case by the usual argument with the one-point compactification.

In the isomorphism in Prop. 4 the left hand side is acted upon by the complexified representation ring $R(G)$ c. The right hand side carries an obvious action by the ring $C l^{\infty}(G)$ of locally constant class functions which is a subring of $\Gamma(G, \mathbb{C})$ fixed by the adjoint action of $G$. Taking traces induces an isomorphism of rings

$$
\operatorname{tr}: R(G) \stackrel{\cong}{\cong} C l^{\infty}(G)
$$

The above proof actually shows that by identifiying the two rings in this way the isomorphism in Prop. 4 becomes an isomorphism of modules over these rings.

\section{Corollary 5:}

There is a natural Chern character isomorphism

$$
K K_{G}^{*}(Y, X) \stackrel{\cong}{\cong} \bigoplus_{j \in \mathbb{Z}} H_{G, c}^{*+2 j}(Y, X)
$$

Proof: By combining Prop. 4 with the ordinary Chern character isomorphism we obtain a natural isomorphism

$$
K K_{G}^{*}(Y, X) \stackrel{\cong}{\longrightarrow} \operatorname{Hom}_{\operatorname{Mod}(\Gamma(G, \mathbb{C}), G)}\left(\underset{i \in \mathbf{Z}}{\oplus} H^{*+2 i}(\hat{Y}, \mathbb{C}), \underset{j \in \mathbf{Z}}{\oplus} H_{c}^{*+2 j}(\hat{X}, \mathbb{C})\right)
$$

which has to be understood in the $\mathbb{Z} / 2 \mathbb{Z}$-graded sense, of course. The right hand side is equal to

$$
\operatorname{Hom}_{\operatorname{Mod}(\Gamma(G, \mathbb{C}), G)}\left(\underset{q \in \mathbf{Z}}{\oplus} H^{q}(\hat{Y}, \mathbb{C}), \underset{j \in \mathbf{Z}}{\oplus} H_{c}^{*+q+2 j}(\hat{X}, \mathbb{C})\right)
$$

Since the cohomology of $\hat{Y}$ is finitely generated over the ring $\Gamma(G, \mathbb{C})$ ( to see this use a decomposition of the form $H^{*}(\hat{Y}, \mathbb{C})=\oplus_{g \in G / N} \Gamma(g H, \mathbb{C}) \otimes H^{*}\left(Y^{g}, \mathbb{C}\right)$ where $N \subseteq G$ is some open subgroup acting trivially on $Y$ ) the second direct sum commutes with the Hom-functor so that our right hand term becomes

$$
\bigoplus_{j \in \mathbf{Z}} \prod_{q \in \mathbf{Z}} \operatorname{Hom}_{\operatorname{Mod}(\Gamma(G, \mathbb{C}), G)}\left(H^{q}(\hat{Y}, \mathbb{C}), H_{c}^{q+*+2 j}(\hat{X}, \mathbb{C})\right)
$$

which according to the last formula in section 1.E is the same as $\underset{j \in \mathbf{Z}}{\oplus} H_{G, c}^{*+2 j}(Y, X)$. 


\section{Representability of bivariant cohomology}

In this final section we will complement the discussion of our bivariant cohomology theory by showing that it has a homotopy theoretic description. Elaborating on $[\mathrm{Hub}]$ we begin by expressing the cohomology of the constant sheaf on a space in a functorial way as the homotopy of a certain spectrum of mapping spaces.

For any two Hausdorff spaces $X$ and $Y$ we let $\operatorname{Map}(X, Y)$ be the space of all continuous maps from $X$ to $Y$ equipped with the compact-open topology; it is Hausdorff again. If $Y$ is pointed then $\operatorname{Map}(X, Y)$ is pointed by the constant map from $X$ to the distinguished point of $Y$. If $Y$ is a topological abelian group then $\operatorname{Map}(X, Y)$ in a natural way is a topological abelian group, too. Clearly

$$
\operatorname{Map}(., Y): U \longmapsto \operatorname{Map}(U, Y)
$$

is a presheaf of topological spaces on $X$.

\section{Lemma 1:}

If $X$ is locally compact then $\operatorname{Map}(., Y)$ is a sheaf.

Proof: The assertion says that whenever $U$ and $U_{i}$, for $i \in I$, are open subsets in $X$ such that $U=\bigcup_{i \in I} U_{i}$ then the commutative diagram of restriction maps

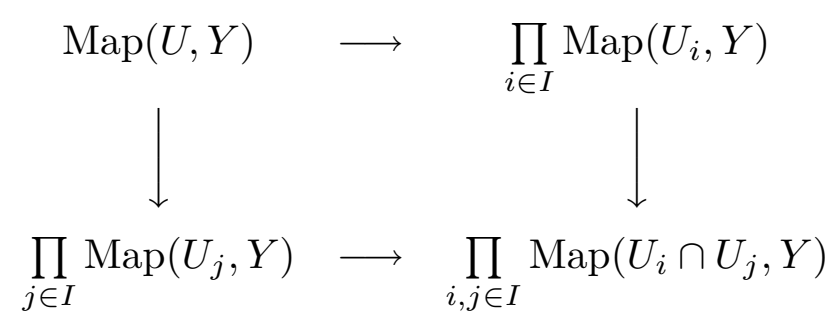

is a cartesian diagram of topological spaces. For trivial reasons it is cartesian as a diagram of sets. Hence it suffices to show that the injective and continuous restriction map

$$
\operatorname{Map}(U, Y) \longrightarrow \prod_{i \in I} \operatorname{Map}\left(U_{i}, I\right)
$$

actually is a homeomorphism onto its image. Let $A \subseteq U$ be a compact and $V \subseteq Y$ be an open subset and consider the open subset $M(A, V):=\{f \in$ $\operatorname{Map}(U, Y): f(A) \subseteq V\}$ in the left hand side. We find finitely many indices $i_{1}, \ldots, i_{n} \in I$ such that $A \subseteq U_{i_{1}} \cup \ldots \cup U_{i_{n}}$. For any point $x \in A$ choose a compact neighbourhood $W_{x}$ of $x$ in some $U_{i_{\nu}}$. Finitely many of those $W_{x}$ cover $A$. Grouping those together appropriately we find compact subsets $A_{\nu} \subseteq U_{i_{\nu}}$ such that $A=A_{1} \cup \ldots \cup A_{n}$. The open subset $M\left(A_{1}, V\right) \times \ldots \times M\left(A_{n}, V\right) \times$ $\prod_{i \neq i_{\nu}} \operatorname{Map}\left(U_{i}, Y\right)$ in the right hand side has $M(A, V)$ as its preimage in the left hand side. 
Next we discuss the stalk

$$
\operatorname{Map}_{x}(X, Y):=\underset{x \in U}{\underset{x \in U}{\longrightarrow} \operatorname{Map}}(U, Y)
$$

of the presheaf $\operatorname{Map}(., Y)$ in the point $x \in X$; the inductive limit of topological spaces is formed over all open neighbourhoods $U$ of $x$ in $X$. There are the two obvious maps

$$
\begin{aligned}
\operatorname{ev}_{x}: \operatorname{Map}_{x}(X, Y) & \longrightarrow Y \\
f & \longmapsto f(x)
\end{aligned}
$$

and

$$
\begin{aligned}
& \text { con }: Y \longrightarrow \operatorname{Map}_{x}(X, Y) \\
& y \longmapsto \text { constant map } \\
& \text { with value } y \text {. }
\end{aligned}
$$

Both maps are continuous satisfying ev $x_{x} \circ \operatorname{con}=i d_{Y}$.

\section{Lemma 2:}

Assume that

- $X$ is locally compact and

- the diagonal in $Y \times Y$ is a deformation retract of one of its open neighbourhoods; then con o $\mathrm{ev}_{x}$ is homotopic to the identity.

Proof: Let $W \subseteq Y \times Y$ be an open neighbourhood of the diagonal $\Delta$ and let $h: W \times[0,1] \longrightarrow W$ be a continuous map such that $h(., 1)=i d_{W}$ and $h(., 0)$ is a retraction of $W$ to $\Delta$. The canonical map

$$
\begin{aligned}
\operatorname{Map}([0,1], Y) & \longrightarrow Y \times Y \\
\omega & \longmapsto(\omega(0), \omega(1))
\end{aligned}
$$

is a fibration ([Spa] 2.8.3); over the diagonal $\Delta$ it has the obvious section $s_{\Delta}$ which maps a point $y \in Y$ to the constant path with value $y$. Applying now the homotopy lifting property of a fibration to the diagram

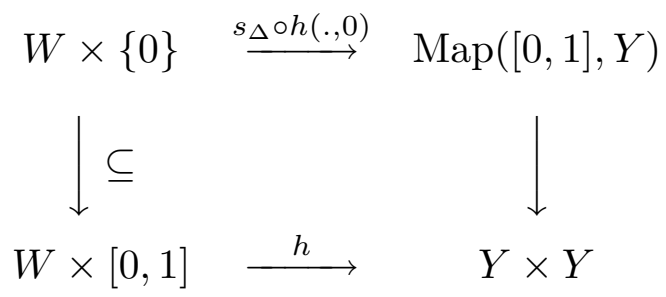

we obtain a continuous map $s: W \times[0,1] \longrightarrow \operatorname{Map}([0,1], Y)$ such that $s(., 1)$ is a section of the above fibration over $W$. We view this section as a continuous 
map $H: W \times[0,1] \longrightarrow Y$ such that $H\left(y, y^{\prime}, 0\right)=y$ and $H\left(y, y^{\prime}, 1\right)=y^{\prime}$. With this map $H$ we can now construct the required homotopy

$$
K:[0,1] \times \underset{x \in U}{\lim } \operatorname{Map}(U, Y) \longrightarrow \underset{x \in U}{\longrightarrow} \operatorname{\operatorname {lim}} \operatorname{Map}(U, Y)
$$

in the following way. Given a $f \in \operatorname{Map}(U, Y)$ let $U(f x):=\left\{x^{\prime} \in U:\left(f(x), f\left(x^{\prime}\right)\right)\right.$ $\in W\}$; this is a possibly smaller open neighbourhood of $x$ such that

$$
\begin{aligned}
f_{t}: U(f x) & \longrightarrow Y \\
x^{\prime} & \longmapsto H\left(f(x), f\left(x^{\prime}\right), t\right)
\end{aligned}
$$

is a well defined continuous map for any $t \in[0,1]$. We put $K(t, f):=f_{t}$. By construction we have $K(1,)=.i d$ and $K(0,)=$. con $\circ \mathrm{ev}_{x}$. It therefore remains to show that $K$ is continuous. For this we consider the subspace

$$
\operatorname{Map}^{\#}(U, Y):=\{f \in \operatorname{Map}(U, Y):\{f(x)\} \times f(U) \subseteq W\} .
$$

We want to show that the obvious continuous map

$$
\underset{x \in U}{\lim } \operatorname{Map}^{\#}(U, Y) \longrightarrow \underset{x \in U}{\longrightarrow} \lim \operatorname{Map}(U, Y)
$$

is a homeomorphism. An inverse map is given by associating with $f \in \operatorname{Map}(U$, $Y)$ the restriction $f \mid U(f x) \in \operatorname{Map}^{\#}(U(f x), Y)$. To see that the so defined map is continuous consider a net $\left\{f_{i}\right\}_{i \in I}$ in $\operatorname{Map}(U, Y)$ which converges to $f \in$ $\operatorname{Map}(U, Y)$. By making the open neighbourhood $U$ of $x$ smaller and by passing to a subset we have to achieve that the net $\left\{f_{i}\right\}$ as well as its limit $f$ lie in $\operatorname{Map}^{\#}(U, Y)$. By a first shrinking of $U$ we may assume that $U=U(f x)$. Next we choose an open neighbourhood $V$ of $f(x)$ in $Y$ such that $V \times V \subseteq W$. By a further shrinking we may assume that $f(U) \subseteq V$. Now we let $A$ be a compact neighbourhood of $x$ in $U$. Then $M(A, V)$ is an open neighbourhood of $f$ in $\operatorname{Map}(U, Y)$ and hence contains a subnet of $\left\{f_{i}\right\}$. If we finally replace $U$ by the interior of $A$ then $f$ as well as that subnet lie in $\operatorname{Map}^{\#}(U, Y)$.

Using that homeomorphism the continuity of $K$ reduces to the continuity of the map

$$
\begin{aligned}
& {[0,1] \times \operatorname{Map}_{(t, f)}^{\#}(U, Y) } \longrightarrow \\
& \operatorname{Map}(U, Y)
\end{aligned}
$$

which is the composite of the two obviously continuous maps

$$
\operatorname{Map}(U, W \times[0,1]) \stackrel{H \circ}{\longrightarrow} \operatorname{Map}(U, Y)
$$

and

$$
\begin{aligned}
{[0,1] \times \operatorname{Map}(U, W) } & \longrightarrow \\
(t, f) & \operatorname{Map}(U, W \times[0,1]) \\
& {\left[x^{\prime} \mapsto\left(f\left(x^{\prime}\right), t\right)\right] }
\end{aligned}
$$


and the third map

$$
\text { id } \times j:[0,1] \times \operatorname{Map}^{\#}(U, Y) \longrightarrow[0,1] \times \operatorname{Map}(U, W)
$$

where $j$ is the restriction to subspaces of the continuous map

$$
\begin{aligned}
& \operatorname{Map}(U, Y) \quad \longrightarrow \quad \operatorname{Map}(U, Y \times Y) \\
& f \longmapsto\left[x^{\prime} \mapsto\left(f(x), f\left(x^{\prime}\right)\right)\right] .
\end{aligned}
$$

\section{Proposition 3:}

It $X$ is locally compact and $Y$ is the topological realization of a countable simplicial set then the map

$$
Y \stackrel{\text { con }}{\longrightarrow} \operatorname{Map}_{x}(X, Y)
$$

is a homotopy equivalence for any $x \in X$.

Proof: The countability assumption guarantuees that $Y \times Y$ with the product topology is the topological realization of the corresponding product of simplicial sets ([LW] II.5.2 and III.5.2). The diagonal in $Y \times Y$ then (as the realization of the simplicial set diagonal) is a subcomplex of a CW-complex and hence a (strong) deformation retract of some open neighbourhood ([LW] II.6.1). The assertion therefore is a special case of the previous lemma.

We will actually need a variant of the latter fact.

\section{Corollary 4:}

Under the same assumptions as in the previous proposition the natural maps between homotopy sets

$$
\pi_{n}(Y, *) \stackrel{\cong}{\underset{x \in U}{\longrightarrow}} \lim _{n}(\operatorname{Map}(U, Y), \operatorname{con}(*)) \stackrel{\cong}{\longrightarrow} \pi_{*}\left(\operatorname{Map}_{x}(X, Y), \operatorname{con}(*)\right)
$$

are bijections for any $x \in X$ and any $n \geq 0$.

Proof: According to Prop. 3 the composed map is a bijection. Hence it suffices to show that the first map is surjective. Fix an open neighbourhood $U$ of $x$ and a pointed continuous map $F: S^{n} \longrightarrow \operatorname{Map}(U, Y)$ from the $n$-sphere $S^{n}$ into $\operatorname{Map}(U, Y)$. Equivalently we may view $F$ as a map $f \in \operatorname{Map}\left(U, \operatorname{Map}_{*}\left(S^{n}, Y\right)\right)$; here $\operatorname{Map}_{*}\left(S^{n}, Y\right) \subseteq \operatorname{Map}\left(S^{n}, Y\right)$ denotes the subspace of base point preserving maps. Since $Y$ satisfies the second assumption of Lemma 2 so also does $\operatorname{Map}_{*}\left(S^{n}, Y\right)$ : One easily checks that if $Y$ is a strong deformation retract of 
its open neighbourhood $W \subseteq Y \times Y$ then $\operatorname{Map}_{*}\left(S^{n}, Y\right)$ is a (strong) deformation retract of its open neighbourhood $\operatorname{Map}_{*}\left(S^{n}, W\right) \subseteq \operatorname{Map}_{*}\left(S^{n}, Y \times Y\right)=$ $\operatorname{Map}_{*}\left(S^{n}, Y\right) \times \operatorname{Map}_{*}\left(S^{n}, Y\right)$. Replacing $U$ by $V:=U(f x)$ the proof of Lemma 2 then provides us with a continuous map

$$
L:[0,1] \longrightarrow \operatorname{Map}\left(V, \operatorname{Map}_{*}\left(S^{n}, Y\right)\right)
$$

such that $L(0)=\operatorname{con}(f(x))$ and $L(1)=f \mid V$. This map can be reinterpreted as a pointed homotopy between $S^{n} \stackrel{F}{\longrightarrow} \operatorname{Map}(U, Y) \longrightarrow \operatorname{Map}(V, Y)$ and $S^{n} \stackrel{f(x)}{\longrightarrow}$ $Y \stackrel{\text { con }}{\longrightarrow} \operatorname{Map}(V, Y)$. In other words the homotopy class of $F$ in $\pi_{n}(\operatorname{Map}(V, Y)$, $\operatorname{con}(*))$ is the image of the homotopy class of $f(x)$ in $\pi_{n}(Y, *)$.

\section{Lemma 5:}

If $X$ is locally compact and $Y$ is contractible as well as a topological abelian group then the sheaf $\operatorname{Map}^{d}(., Y)$ of abelian groups on $X$ which underlies $\operatorname{Map}(., Y)$ is c-soft.

Proof: Since any compact subset of $X$ has a fundamental system of compact neighbourhoods this is the same argument as in the proof of [Hub] 3.2.

We now will use these "mapping sheaves" to construct an explicit resolution of the in both directions unbounded complex of sheaves

$$
\mathbb{Q}_{X}^{\text {per }}: \ldots \longrightarrow \mathbb{Q}_{X} \longrightarrow 0 \longrightarrow \mathbb{Q}_{X} \longrightarrow \ldots
$$

on $X$ which has the constant sheaf $\mathbb{Q}_{X}$, resp. the zero sheaf, in every even, resp. odd, degree. If $X=p t$ is a point then $\mathbb{Q}_{p t}^{\text {per }}$ is a complex of $\mathbb{Q}$-vector spaces and we simply write $\mathbb{Q}^{\text {per }}:=\mathbb{Q}_{p t}^{\text {per }}$. Applying the Dold-Kan functor we obtain the spectrum of simplicial abelian sheaves $D K\left(\mathbb{Q}_{X}^{\text {per }}\right)$ on $X$ as well as the spectrum of countable simplicial abelian groups $D K\left(\mathbb{Q}^{\text {per }}\right)$. The topological realization $\left|D K\left(\mathbb{Q}_{X}^{\text {per }}\right)\right|$ of the former is a presheaf of spectra of pointed topological spaces on $X$. The topological realization $\left|D K\left(\mathbb{Q}^{\text {per }}\right)\right|$ of the latter is a spectrum of topological abelian groups.

At this point we should say that for us a spectrum $Z$ of pointed topological spaces is a collection of pointed Hausdorff spaces $Z^{0}, Z^{1}, \ldots, Z^{n}, \ldots$ together with pointed continuous maps $S Z^{n} \longrightarrow Z^{n+1}$ (or equivalently, by adjunction, $\left.Z^{n} \longrightarrow \Omega Z^{n+1}\right)$ for any $n \geq 0$. Here $S$ and $\Omega$ denote the suspension and loop space functor on pointed spaces, respectively. Assume that each $Z^{n}$ is a topological abelian group (viewed as pointed by the zero element). Then the $\Omega Z^{n}$ in a natural way are topological abelian groups, too, and $Z$ is called a spectrum of topological abelian groups if the maps $Z^{n} \longrightarrow \Omega Z^{n+1}$ are group homomorphisms. Concerning maps $f: Z \longrightarrow Z^{\prime}$ between spectra we adopt the 
naive point of view that $f$ is a collection of pointed continuous maps $f^{n}: Z^{n} \longrightarrow$ $Z^{\prime n}$ such that the diagrams

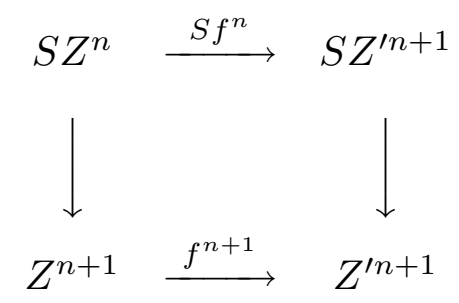

are commutative for any $n \geq 0$. Moreover $f$ is called a weak equivalence if each $f^{n}$ is one.

Note that if $X$ is a locally compact and $Y$ an arbitrary Hausdorff space then the obvious map

$$
\Omega \operatorname{Map}(X, Y) \stackrel{\sim}{\longrightarrow} \operatorname{Map}(X, \Omega Y)
$$

is a homeomorphism. This allows us to define, for any locally compact space $X$ and any spectrum $Z$, the function $\operatorname{spectrum} \operatorname{Map}(X, Z)$ by $\operatorname{Map}(X, Z)^{n}:=$ $\operatorname{Map}\left(X, Z^{n}\right)$ with the maps $\operatorname{Map}\left(X, Z^{n}\right) \longrightarrow \Omega \operatorname{Map}\left(X, Z^{n+1}\right)$ arising from composition with the map $Z^{n} \longrightarrow \Omega Z^{n+1}$. The homotopy groups of the function spectrum are

$$
\pi_{r}(\operatorname{Map}(X, Z))=\underset{n>-r}{\lim } \pi_{n+r}\left(\operatorname{Map}\left(X, Z^{n}\right)\right)=\underset{n>-r}{\lim }\left[S^{n+r}(X \dot{\cup}\{*\}), Z^{n}\right]_{*}
$$

Here, $[., .]_{*}$ stands for the pointed homotopy classes of maps between two pointed spaces. If $Z$ is a spectrum of topological abelian groups so, too, is $\operatorname{Map}(X, Z)$.

For the convenience of the reader we will recall in an appendix to this section the details about the Dold-Kan correspondence.

For the rest of this section let $X$ be a fixed locally compact space. From the spectrum $\left|D K\left(\mathbb{Q}^{\text {per }}\right)\right|$ we obtain the presheaf of spectra of pointed topological spaces (in fact topological abelian groups) on $X$ given by

$$
\operatorname{Map}\left(.,\left|D K\left(\mathbb{Q}^{\text {per }}\right)\right|\right): U \longmapsto \operatorname{Map}\left(U,\left|D K\left(\mathbb{Q}^{\text {per }}\right)\right|\right)
$$

The basis of our construction is the existence of a natural map

$$
\varepsilon:\left|D K\left(\mathbb{Q}_{X}^{\text {per }}\right)\right| \longrightarrow \operatorname{Map}\left(.,\left|D K\left(\mathbb{Q}^{\text {per }}\right)\right|\right)
$$

whose definition is very simple. To begin let us fix an open subset $U \subseteq X$. Recall that the space of sections $\mathbb{Q}_{X}(U)$ is the space of locally constant $\mathbb{Q}$ valued functions on $U$. Hence for any point $x \in U$ evaluating a function at $x$ 
defines a homomorphism of complexes $e v_{x}: \mathbb{Q}_{X}^{\text {per }}(U) \longrightarrow \mathbb{Q}^{\text {per }}$. By functoriality we obtain from this the maps

$$
\begin{aligned}
\varepsilon_{U}^{n}:\left|D K\left(\mathbb{Q}_{X}^{\text {per }}(U)\right)\right|^{n} \times U & \longrightarrow\left|D K\left(\mathbb{Q}^{\text {per }}\right)\right|^{n} \\
(a, x) & \longmapsto\left|D K\left(e v_{x}\right)\right|^{n}(a)
\end{aligned}
$$

for any $n \geq 0$. We claim that $\varepsilon_{U}^{n}$ is continuous. Using the definition of the topological realization of a simplicial set (as well as the fact that $U$ is locally compact) this reduces to the statement that the maps

$$
\begin{aligned}
D K\left(\mathbb{Q}_{X}^{\mathrm{per}}(U)\right)_{m}^{n} \times U & \longrightarrow D K\left(\mathbb{Q}^{\mathrm{per}}\right)_{m}^{n} \\
(s, x) & \longmapsto D K\left(e v_{x}\right)_{m}^{n}(s)
\end{aligned}
$$

are continuous where the subscript $m$ indicates the set of $m$-simplices in the respective simplicial set equipped with the discrete topology. The definition of the functor $D K$ tells us that the set (or rather group) of $m$-simplices of the simplicial abelian groups under consideration is given as a certain finite direct sum of copies of $\mathbb{Q}_{X}(U)$ and $\mathbb{Q}$, respectively. Hence we are reduced to the continuity of the evaluation pairing $\mathbb{Q}_{X}(U) \times U \longrightarrow \mathbb{Q}$ which is trivial.

This establishes our claim and shows that $\varepsilon_{U}^{n}$ induces a pointed continuous map

$$
\left|D K\left(\mathbb{Q}_{U}^{\text {per }}(U)\right)\right|^{n} \longrightarrow \operatorname{Map}\left(U,\left|D K\left(\mathbb{Q}^{\text {per }}\right)\right|^{n}\right)
$$

It is clear that these maps combine into a map $\varepsilon$ of presheaves of spectra.

\section{Lemma 6:}

The map

$$
\varepsilon:\left|D K\left(\mathbb{Q}_{X}^{\text {per }}\right)\right| \longrightarrow \operatorname{Map}\left(.,\left|D K\left(\mathbb{Q}^{\text {per }}\right)\right|\right)
$$

is stalkwise a weak equivalence of spectra of topological spaces.

Proof: We have $\left|D K\left(\mathbb{Q}_{X}^{\text {per }}\right)\right|^{n}=\left|D K\left(\mathbb{Q}_{X}^{(n)}\right)\right|$ for a certain shifted truncation $\mathbb{Q}_{X}^{(n)}$ of the complex $\mathbb{Q}_{X}^{\text {per }}$ and similarly $\left|D K\left(\mathbb{Q}^{\text {per }}\right)\right|^{n}=\left|D K\left(\mathbb{Q}^{(n)}\right)\right|$ (compare the appendix). The functor $|$.$| of topological realization as a left adjoint as well as the$ functor $D K$ as a category equivalence commute with inductive limits. Moreover the filtered inductive limit of abelian groups coincides with the inductive limit of the underlying sets. Hence the stalks on the left hand side can be computed as follows:

$$
\left.\underset{x \in U}{\lim }\left|D K\left(\mathbb{Q}_{X}^{\text {per }}(U)\right)\right|^{n}=\mid D K \underset{x \in U}{\left(\lim _{x \in U}\right.} \mathbb{Q}_{X}^{(n)}(U)\right)|=| D K\left(\mathbb{Q}^{(n)}\right) \mid
$$


The assertion therefore follows by applying Prop. 3 to the space $Y:=\mid D K$ $\left(\mathbb{Q}^{(n)}\right) \mid$.

The map $\varepsilon$ can be turned into a resolution of the complex of sheaves $\mathbb{Q}_{X}^{\text {per }}$ in the following way. First we recall that the realization functor $|$.$| from pointed$ simplicial sets into pointed Hausdorff spaces has a right adjoint - the singular complex functor Sin defined by

$$
(\operatorname{Sin} Y)_{m}:=\text { set of all continuous maps from } \Delta^{m} \text { into } Y
$$

where $\Delta^{m}$ denotes the geometrical $m$-simplex and where the face and degeneracy maps come from the structure of $\left(\Delta^{m}\right)_{m}$ as a cosimplicial space. Moreover the adjunction maps $S$. $\longrightarrow \operatorname{Sin} \mid S$. $\mid$ and $|\operatorname{Sin} Y| \longrightarrow Y$ are weak homotopy equivalences. The following further properties are straightforward to check:

- If $Y$ is a topological abelian group then $\operatorname{Sin} Y$ is a simplicial abelian group.

- There is a natural isomorphism $\operatorname{Sin}(\Omega Y) \cong \omega(\operatorname{Sin} Y)$ (corresponding under adjunction to the isomorphism $\mid \Sigma S$. $|\cong S| S$. $\mid$ in the appendix).

This allows us to define, for any spectrum of topological abelian groups $Z$, the spectrum of simplicial abelian groups $\operatorname{Sin} Z:=\left(\operatorname{Sin} Z^{n}\right)_{n}$ in the sense that the structure maps of the spectrum $\operatorname{Sin} Z^{n} \longrightarrow \omega\left(\operatorname{Sin} Z^{n+1}\right)$ are not required to be isomorphisms (as we do in the appendix below). Nevertheless the normalization functor still makes sense and produces a complex $N(\operatorname{Sin} Z)$ of abelian groups with the property that

$$
\pi_{*}(Z)=h_{*}(N(\operatorname{Sin} Z)) .
$$

Hence, by applying adjunction and normalization sectionwise, we obtain from $\varepsilon$ a homomorphism of complexes of presheaves of $\mathbb{Q}$-vector spaces

$$
\varepsilon: \mathbb{Q}_{X}^{\text {per }} \longrightarrow N\left(\operatorname{Sin} \operatorname{Map}\left(.,\left|D K\left(\mathbb{Q}^{\text {per }}\right)\right|\right)\right)
$$

on $X$. The left hand side, of course, is a complex of sheaves. Concerning the right hand side note first of all that $\operatorname{Map}\left(U,\left|D K\left(\mathbb{Q}^{\text {per }}\right)\right|^{n}\right)$ is a topological abelian group and is, with varying $U$, a sheaf according to Lemma 1 . It easily follows that $\operatorname{Sin} \operatorname{Map}\left(.,\left|D K\left(\mathbb{Q}^{\text {per }}\right)\right|^{n}\right)$ actually is a simplicial sheaf and that consequently $N\left(\operatorname{Sin} \operatorname{Map}\left(.,\left|D K\left(\mathbb{Q}^{\text {per }}\right)\right|^{n}\right)\right)$ is a complex of sheaves on $X$. Since an inductive limit of sheaves cannot be performed sectionwise we define

$$
\begin{aligned}
H R\left(\mathbb{Q}_{X}^{\text {per }}\right) & :=\text { sheafification of } N\left(\operatorname{Sin} \operatorname{Map}\left(.,\left|D K\left(\mathbb{Q}^{\text {per }}\right)\right|\right)\right) \\
& =\text { sheaf direct limit of } N\left(\operatorname{Sin} \operatorname{Map}\left(.,\left|D K\left(\mathbb{Q}^{\text {per }}\right)\right|^{n}\right)\right)[n]
\end{aligned}
$$

( $H R$ stands for "homotopical resolution") and obtain from $\varepsilon$ the homomorphism of complexes of sheaves of $\mathbb{Q}$-vector spaces

$$
\text { res : } \mathbb{Q}_{X}^{\text {per }} \longrightarrow H R\left(\mathbb{Q}_{X}^{\text {per }}\right)
$$


on $X$.

\section{Remark 7:}

Passing to global sections on a compact space commutes with inductive limits of sheaves. We therefore have

$$
\begin{aligned}
h_{*}\left(H R\left(\mathbb{Q}_{X}^{\text {per }}\right) \cdot(X)\right) & =\underset{n \geq 0}{\lim } h_{*}\left(N\left(\operatorname{Sin} \operatorname{Map}\left(X,\left|D K\left(\mathbb{Q}^{\text {per }}\right)\right|^{n}\right)\right)[n]\right) \\
& =\underset{n \geq 0}{\underset{n}{\longrightarrow}} \pi_{*+n}\left(\operatorname{Map}\left(X,\left|D K\left(\mathbb{Q}^{\text {per }}\right)\right|^{n}\right)\right) \\
& =\pi_{*}\left(\operatorname{Map}\left(X,\left|D K\left(\mathbb{Q}^{\text {per }}\right)\right|\right)\right)
\end{aligned}
$$

provided $X$ is compact.

\section{Lemma 8:}

res is a quasi-isomorphism.

Proof: Applying Cor. 4 we obtain

$$
\begin{aligned}
& h_{*}\left(\underset{x \in U}{\lim } \mathbb{Q}_{X}^{\text {per }}(U)\right)=h_{*}\left(\mathbb{Q}^{\text {per }}\right)=\pi_{*}\left(\left|D K\left(\mathbb{Q}^{\text {per }}\right)\right|\right) \\
& =\underset{x \in U}{\lim \pi_{*}}\left(\operatorname{Map}\left(U,\left|D K\left(\mathbb{Q}^{\text {per }}\right)\right|\right)\right) \\
& =\underset{x \in U}{\lim } h_{*}\left(N\left(\operatorname{Sin} \operatorname{Map}\left(U,\left|D K\left(\mathbb{Q}^{\text {per }}\right)\right|\right)\right)\right) \\
& \left.=h_{*} \underset{x \in U}{\lim _{\longrightarrow}} H R\left(\mathbb{Q}_{X}^{\text {per }}\right)(U)\right) .
\end{aligned}
$$

\section{Lemma 9:}

The complex $H R\left(\mathbb{Q}_{X}^{\mathrm{per}}\right)$ consists of $\mathrm{c}$-soft sheaves.

Proof: For the $r$-th sheaf in the complex $H R\left(\mathbb{Q}_{X}^{\text {per }}\right)$ we have the formula

$$
\begin{aligned}
& H R\left(\mathbb{Q}_{X}^{\text {per }}\right)_{r} \quad \underset{n}{\underset{n}{\lim }} N\left(\operatorname{Sin} \operatorname{Map}\left(.,\left|D K\left(\mathbb{Q}^{\text {per }}\right)\right|^{n}\right)\right)[n]_{r} \\
& =\underset{n}{\lim } N\left(\operatorname{Sin} \operatorname{Map}\left(.,\left|D K\left(\mathbb{Q}^{\text {per }}\right)\right|^{n}\right)\right)_{n+r} \\
& =\underset{n}{\lim } \operatorname{Map}_{*}^{d}\left(\Delta^{n+r} / \Lambda^{n+r}, \operatorname{Map}\left(.,\left|D K\left(\mathbb{Q}^{\text {per }}\right)\right|^{n}\right)\right) \\
& =\underset{n}{\lim } \operatorname{Map}^{d}\left(., \operatorname{Map}_{*}\left(\Delta^{n+r} / \Lambda^{n+r},\left|D K\left(\mathbb{Q}^{\text {per }}\right)\right|^{n}\right)\right)
\end{aligned}
$$


where $\Lambda^{m} \subseteq \Delta^{m}$ denotes the subcomplex generated by all except the last codimension 1 faces. The quotient $\Delta^{m} / \Lambda^{m}$ is contractible as a pointed space. It easily follows that $\operatorname{Map}_{*}\left(\Delta^{m} / \Lambda^{m}, Y\right)$ is contractible for any pointed Hausdorff space $Y$. Hence Lemma 5 implies that $H R\left(\mathbb{Q}_{X}^{\text {per }}\right)$ is an inductive limit of $c$-soft sheaves and therefore is $c$-soft.

Let $t_{\leq n} D$., resp. $s_{\leq n} D$., be the truncation functor on complexes which is defined by

$$
t_{\leq n} D .:=\left[\ldots \longrightarrow 0 \longrightarrow D_{n} / \operatorname{im}\left(d_{n+1}\right) \longrightarrow D_{n-1} \longrightarrow \ldots\right]
$$

resp. by

$$
s_{\leq n} D .:=\left[\ldots \longrightarrow 0 \longrightarrow D_{n} \longrightarrow D_{n-1} \longrightarrow \ldots\right],
$$

with degrees unchanged. They are related by the exact sequence

$$
0 \longrightarrow \operatorname{im}\left(d_{n+1}\right)[-n] \longrightarrow s_{\leq n} D . \longrightarrow t_{\leq n} D . \longrightarrow 0
$$

The functor $t_{\leq n}$ preserves quasi-isomorphisms. The complexes $s_{\leq n} D$. for $n \geq 0$ form an inductive system whose limit is $D$. .

We are interested here in the inductive system of homomorphisms of complexes

$$
s_{\leq n} \text { res }: s_{\leq n} \mathbb{Q}_{X}^{\text {per }} \longrightarrow s_{\leq n} H R\left(\mathbb{Q}_{X}^{\text {per }}\right)
$$

for $n \geq 0$. The cohomology of the left hand side clearly is equal to

$$
H_{c}^{*}\left(X, s_{\leq n} \mathbb{Q}_{X}^{\mathrm{per}}\right)=\underset{\substack{j \in \mathrm{Z} \\ 2 j \leq n}}{\bigoplus} H_{c}^{*+2 j}(X, \mathbb{Q})
$$

(We stick to the convention that hypercohomology is a functor on cohomological complexes. This means that we silently change the indexing of our complexes from the lower to the upper indexing before we take hypercohomology!) By Lemma 9 the right hand side is a complex of $c$-soft sheaves which in cohomological indexing is bounded below. Hence its cohomology, for $*>-n$, is

$$
H_{c}^{*}\left(X, s_{\leq n} H R\left(\mathbb{Q}_{X}^{\text {per }}\right)\right)=h_{-*}\left(\Gamma_{c}\left(X, H R\left(\mathbb{Q}_{X}^{\text {per }}\right)\right)\right) .
$$

In the limit with respect to $n$ we therefore obtain a natural map

$$
\text { res : } \bigoplus_{j \in \mathbf{Z}} H_{c}^{*+2 j}(X, \mathbb{Q}) \longrightarrow h_{*}\left(\Gamma_{c}\left(X, H R\left(\mathbb{Q}_{X}^{\text {per }}\right)\right)\right)
$$

which for a compact space $X$, by Remark 7 , can be seen as a natural map

$$
\text { res : } \oplus_{j \in \mathbf{Z}} H^{*+2 j}(X, \mathbb{Q}) \longrightarrow \pi_{*}\left(\operatorname{Map}\left(X,\left|D K\left(\mathbb{Q}^{\text {per }}\right)\right|\right)\right)
$$




\section{Proposition 10:}

If $X$ is compact then the map

$$
\text { res : } \underset{j \in \mathbf{Z}}{\bigoplus} H^{*+2 j}(X, \mathbb{Q}) \stackrel{\cong}{\longrightarrow} \pi_{*}\left(\operatorname{Map}\left(X,\left|D K\left(\mathbb{Q}^{\text {per }}\right)\right|\right)\right)
$$

is an isomorphism.

Proof: For the complex $\mathbb{Q}_{X}^{\text {per }}$ the two truncations $t_{\leq n} \mathbb{Q}_{X}^{\text {per }}$ and $s_{\leq n} \mathbb{Q}_{X}^{\text {per }}$ coincide. We therefore have the commutative exact diagram

$$
\begin{aligned}
& s_{\leq n} \mathbb{Q}_{X}^{\text {per }} \stackrel{=}{\longrightarrow} t_{\leq n} \mathbb{Q}_{X}^{\text {per }} \\
& \downarrow s_{\leq n} \text { res } \quad \downarrow t_{\leq n} \text { res } \\
& 0 \longrightarrow \operatorname{im}\left(d_{n+1}\right)[-n] \longrightarrow s_{\leq n} H R\left(\mathbb{Q}_{X}^{\text {per }}\right) \longrightarrow t_{\leq n} H R\left(\mathbb{Q}_{X}^{\text {per }}\right) \longrightarrow 0 \text {. }
\end{aligned}
$$

By Lemma $8, t_{\leq n}$ res is a quasi-isomorphism and hence induces an isomorphism in cohomology. This means that in cohomology we have isomorphisms

$$
H^{*+n}\left(X, \operatorname{im}\left(d_{n+1}\right)\right) \oplus H^{*}\left(X, s_{\leq n} \mathbb{Q}_{X}^{\text {per }}\right) \stackrel{\cong}{\longrightarrow} H^{*}\left(X, s_{\leq n} H R\left(\mathbb{Q}_{X}^{\text {per }}\right)\right)
$$

It remains to show that in the limit with respect to $n$ the first summand on the left hand side disappears. First of all we note that because of Lemma 9 we have

$$
H^{*+n}\left(X, \operatorname{im}\left(d_{n+1}\right)\right)=H^{*+n+1}\left(X, \operatorname{ker}\left(d_{n+1}\right)\right)
$$

provided $*+n>0$. Next we decompose the whole complex $H R\left(\mathbb{Q}_{X}^{\text {per }}\right)$ and hence in particular each sheaf $\operatorname{ker}\left(d_{n}\right)$ into a direct sum. For each $m \in \mathbb{Z}$ let $\mathbb{Q}[m]$ denote the unbounded complex of $\mathbb{Q}$-vector spaces which has $\mathbb{Q}$ in degree $-m$ and has 0 elsewhere. We define the complex $H R\left(\mathbb{Q}_{X}[m]\right)$ of sheaves of $\mathbb{Q}$-vector spaces on $X$ by

$$
H R\left(\mathbb{Q}_{X}[m]\right):=\text { sheafification of } N(\operatorname{Sin} \operatorname{Map}(.,|D K(\mathbb{Q}[m])|)) \text {. }
$$

The obvious identity $\mathbb{Q}^{\text {per }}=\underset{m \in \mathbf{Z}}{\oplus} \mathbb{Q}[2 m]$ induces by functoriality a homomorphism of complexes of sheaves

$$
\underset{m \in \mathbf{Z}}{\oplus} H R\left(\mathbb{Q}_{X}[2 m]\right) \longrightarrow H R\left(\mathbb{Q}_{X}^{\text {per }}\right)
$$

which we claim is an isomorphism. For this we compare the stalks in each degree; fix a degree $r \in \mathbb{Z}$ and a point $x \in X$. Similarly as in the proof of Lemma 9 the stalk of the sheaf $H R\left(\mathbb{Q}_{X}^{\text {per }}\right)_{r}$ in $x$ is given by

$$
\underset{x \in U}{\lim } \underset{\ell}{\lim } \operatorname{Map}_{*}^{d}\left(U_{\ell+r},\left|D K\left(\mathbb{Q}^{\text {per }}\right)\right|^{\ell}\right)
$$


where $U_{\ell+r}:=\left(\Delta^{\ell+r} / \Lambda^{\ell+r} \times U\right) /(* \times U)$. Correspondingly the stalk in $x$ in degree $r$ of the left hand side is equal to

$$
\underset{m \in \mathbf{Z}}{\oplus} \underset{x \in U}{\lim } \underset{\ell}{\lim } \operatorname{Map}_{*}^{d}\left(U_{\ell+r},|D K(\mathbb{Q}[2 m])|^{\ell}\right)
$$

The decomposition $\mathbb{Q}^{\text {per }}=\underset{m \in \mathbf{Z}}{\oplus} \mathbb{Q}[2 m]$ implies, by the basic properties of the functors involved (which we have used already repeatedly), the identity

$$
\left|D K\left(\mathbb{Q}^{\text {per }}\right)\right|^{\ell}=\lim _{m \in \mathbb{N}} \prod_{-m \leq k \leq m}|D K(\mathbb{Q}[2 k])|^{\ell} .
$$

Since $U$ contains a compact neighbourhood of $x$ any continuous map from $U_{\ell+r}$ into the inductive limit on the right hand side has, after shrinking $U$, its image already in some finite product $\prod_{-m \leq k \leq m}|D K(\mathbb{Q}[2 k])|^{\ell}$. This means that the two stalks in question are the same.

With the above isomorphism established, we obtain the decomposition

$$
\operatorname{ker}\left(d_{n}\right)=\underset{m \in \mathbf{Z}}{\oplus} \operatorname{ker}\left(d_{n}^{[2 m]}\right)
$$

where $d_{n}^{[m]}$ denotes the boundary map in the complex $H R\left(\mathbb{Q}_{X}[m]\right)$. Since $X$ is compact we therefore are reduced to showing that $\underset{n}{\lim } H^{*+n}\left(X, \operatorname{ker}\left(d_{n}^{[2 m]}\right)\right)=0$ for each $m \in \mathbb{Z}$. In fact we will show that the sheaf $\operatorname{ker}\left(d_{n}^{[m]}\right)$ is $c$-soft provided $n>-m$. We have

$$
\begin{aligned}
& \operatorname{ker}\left(d_{n}^{[m]}\right)=\underset{\ell}{\lim } \operatorname{Map}_{*}^{d}\left(\Delta^{\ell+n} / \partial \Delta^{\ell+n}, \operatorname{Map}\left(.,|D K(\mathbb{Q}[m])|^{\ell}\right)\right) \\
& =\underset{\ell}{\lim } \operatorname{Map}_{*}^{d}\left(S^{\ell+n}, \operatorname{Map}\left(.,|D K(\mathbb{Q}[m])|^{\ell}\right)\right) \\
& =\underset{\ell}{\lim } \operatorname{Map}^{d}\left(., \operatorname{Map}_{*}\left(S^{\ell+n},|D K(\mathbb{Q}[m])|^{\ell}\right)\right) \\
& =\underset{\ell}{\lim } \operatorname{Map}^{d}\left(., \operatorname{Map}_{*}\left(S^{\ell+n},|D K(\mathbb{Q}[m-\ell])|\right)\right) \\
& =\underset{\ell}{\lim } \operatorname{Map}^{d}\left(., \Omega^{\ell+n}|D K(\mathbb{Q}[m-\ell])|\right) .
\end{aligned}
$$

Using Lemma 5 we see that it is sufficient to check that the space $\Omega^{\ell+n} \mid D K$ $(\mathbb{Q}[m-\ell]) \mid$ is contractible for $n>-m>-\ell$. By its very construction $|D K(\mathbb{Q}[m-\ell])|$ is a $C W$-complex as well as a $K(\mathbb{Q}, \ell-m)$-space (for $\ell>m)$. Its $(\ell+n)$-fold loop space, for $n>-m$, therefore has vanishing homotopy groups. 
Since, according to [Mil], the loop space functor respects the category of spaces which have the homotopy type of a CW-complex we may apply the Whitehead theorem and deduce the contractibility we wanted.

For the rest of this section we assume that the locally compact space $X$ is a $G$-space for the locally compact and totally disconnected group $G$. We need to know that the results established so far hold true in an equivariant way. This actually requires a modification of our homotopical resolution for the following reason. The naturality of this resolution of course implies that $\mathbb{Q}_{X}^{\text {per }} \stackrel{\text { res }}{\longrightarrow} H R\left(\mathbb{Q}_{X}^{\text {per }}\right)$ is $G^{\text {dis }}$-equivariant where $G^{\text {dis }}$ denotes $G$ viewed as a discrete group. But the sheaves $H R\left(\mathbb{Q}_{X}^{\text {per }}\right)_{r}$ in general will not be $G$-equivariant. By applying the functor $(.)^{\text {smooth }}: \operatorname{Sh}_{G}$ dis $(X) \longrightarrow \operatorname{Sh}_{G}(X)$ (compare $[\mathrm{Sch}] \S 1$; in this section the notation refers to sheaves of $\mathbb{Q}$-vector spaces) degreewise to $H R\left(\mathbb{Q}_{X}^{\text {per }}\right)$ we obtain a complex $H R\left(\mathbb{Q}_{X}^{\text {per }}\right)^{\text {smooth }}$ in $\operatorname{Sh}_{G}(X)$. Since the complex $\mathbb{Q}_{X}^{\text {per }}$ consists of $G$-equivariant sheaves the map res factorizes through an equivariant homomorphism of complexes

$$
\operatorname{res}^{\text {smooth }}: \mathbb{Q}_{X}^{\text {per }} \longrightarrow H R\left(\mathbb{Q}_{X}^{\text {per }}\right)^{\text {smooth }}
$$

Let us go step by step through our above results and discuss the corresponding $G$-equivariant versions. For an open subset $U \subseteq X$ and a Hausdorff space $Y$ let $\operatorname{Map}(U, Y)^{\text {smooth }}$ denote the subspace in $\operatorname{Map}(U, Y)$ of all "smooth" continuous maps from $U$ to $Y$. A continuous map $f: U \longrightarrow Y$ is called smooth if there is an open covering $U=\bigcup_{i \in I} U_{i}$ together with open subgroups $H_{i} \subseteq G$ such that, for any $i \in I, U_{i}$ is $H_{i}$-invariant and $f \mid U_{i}$ factorizes through the quotient $H_{i} \backslash U_{i}$. For any point $x \in X$ we define $\operatorname{Map}_{x}(X, Y)^{\text {smooth }}:=\underset{x \in U}{\lim } \operatorname{Map}(U, Y)^{\text {smooth }}$. With proofs analogous to the above ones we then have the following facts:

A) $\operatorname{Map}(., Y)^{\text {smooth }}$ is a sheaf of topological spaces on $X$. If $Y$ is a topological abelian group then the underlying sheaf of abelian groups $\operatorname{Map}^{d}(., Y)^{\text {smooth }}$ is $G$-equivariant.

B) Let $Y$ be the topological realization of a countable simplicial set. Then, for any $x \in X$, the map

$$
Y \stackrel{\text { con }}{\longrightarrow} \operatorname{Map}_{x}(X, Y)^{\text {smooth }}
$$

is a homotopy equivalence.

C) If $Y$ is contractible as well as a topological abelian group then $\operatorname{Map}^{d}($. , $Y)^{\text {smooth }}$ is $c$-soft. (Here we use that any compact subset of $X$ has a fundamental system of open neighbourhoods $U$ each of which is invariant under some compact open subgroup $H \subseteq G$. The argument in [Hub] 3.2 then has to be carried out on an appropriate quotient $H \backslash U$.)

In order to state the equivariant version of Cor. 4 we need further notations. An equivariant neighbourhood of a point $x \in X$ is a pair $(U, H)$ consisting of 
an open neighbourhood $U \subseteq X$ of $x$ and an open subgroup $H \subseteq G$ such that $U$ is $H$-invariant. The set $\mathfrak{U}(x)$ of all equivariant neighbourhoods is a directed set with respect to the partial order

$$
(U, H) \leq\left(U^{\prime}, H^{\prime}\right) \text { if } U \supseteq U^{\prime} \text { and } H \supseteq H^{\prime} \text {. }
$$

For any $(U, H) \in \mathfrak{U}(x)$ we have the subspace $\operatorname{Map}(U, Y)^{H}$ of $H$-invariant maps in $\operatorname{Map}(U, Y)$. There is an obvious continuous bijection

$$
\underset{(U, H) \in \mathfrak{U}(x)}{\lim } \operatorname{Map}(U, Y)^{H} \longrightarrow \operatorname{Map}_{x}(X, Y)^{\text {smooth }}
$$

\section{Lemma 11:}

Let $Y$ be the topological realization of a countable simplicial set; then the natural maps

$$
\begin{aligned}
\pi_{n}(Y, *) & \stackrel{\cong}{\longrightarrow} \lim _{(U, H) \in \mathfrak{U}(x)} \pi_{n}\left(\operatorname{Map}(U, Y)^{H}, \operatorname{con}(*)\right) \\
& \cong \pi_{n}\left(\operatorname{Map}_{x}(X, Y)^{\text {smooth }}, \operatorname{con}(*)\right)
\end{aligned}
$$

are bijections for any $x \in X$ and any $n \geq 0$.

Proof: According to B) the composed map is a bijection. Hence it suffices to show that the first map is surjective. Fix an equivariant neighbourhood $(U, H)$ of $x$ and a pointed continuous map $F: S^{n} \longrightarrow \operatorname{Map}(U, Y)^{H}$. We view the latter as a map $f \in \operatorname{Map}\left(U, \operatorname{Map}_{*}\left(S^{n}, Y\right)\right)^{H}$. The map

$$
L:[0,1] \longrightarrow \operatorname{Map}\left(V, \operatorname{Map}_{*}\left(S^{n}, Y\right)\right)
$$

with $V:=U(f x)$ which we used in the proof of Cor. 4 was of the form

$$
L(t)\left(x^{\prime}\right)=\tilde{H}\left(\left(f(x), f\left(x^{\prime}\right)\right), t\right)
$$

for an appropriate continuous map $\tilde{H}: \operatorname{Map}_{*}\left(S^{n}, W\right) \times[0,1] \longrightarrow \operatorname{Map}_{*}\left(S^{n}, Y\right)$. This shows that all the continuous maps $L(t)$ have the "same" invariance properties with respect to the $H$-action as $f$ does. More precisely, if $\left(U^{\prime}, H^{\prime}\right)$ is an equivariant neighbourhood of $x$ such that $(U, H) \leq\left(U^{\prime}, H^{\prime}\right)$ and $U^{\prime} \subseteq V$ then $L(t) \mid U^{\prime}$ is $H^{\prime}$-invariant for any $t \in[0,1]$. Therefore $L$ can be viewed as a pointed homotopy between $S^{n} \stackrel{F}{\longrightarrow} \operatorname{Map}(U, Y)^{H} \longrightarrow \operatorname{Map}\left(U^{\prime}, Y\right)^{H^{\prime}}$ and $S^{n} \stackrel{f(x)}{\longrightarrow} Y \stackrel{\text { con }}{\longrightarrow} \operatorname{Map}\left(U^{\prime}, Y\right)^{H^{\prime}}$.

We have $\operatorname{Map}(U, \Omega Y)^{\text {smooth }} \subseteq \Omega \operatorname{Map}(U, Y)^{\text {smooth }}$. Hence if $Z$ is a spectrum of pointed topological spaces then $\operatorname{Map}(U, Z)^{\text {smooth }}:=\left(\operatorname{Map}\left(U, Z^{n}\right)^{\text {smooth }}\right)_{n}$ is a subspectrum of $\operatorname{Map}(U, Z)$. In this way we obtain in particular the subpresheaf 
$\operatorname{Map}\left(.,\left|D K\left(\mathbb{Q}^{\text {per }}\right)\right|\right)^{\text {smooth }}$ of $\operatorname{Map}\left(.,\left|D K\left(\mathbb{Q}^{\text {per }}\right)\right|\right)$. The above discussion of the continuity of our map $\varepsilon$ shows that $\varepsilon$ factorizes through a map

$$
\varepsilon^{\text {smooth }}:\left|D K\left(\mathbb{Q}_{X}^{\text {per }}\right)\right| \longrightarrow \operatorname{Map}\left(.,\left|D K\left(\mathbb{Q}^{\text {per }}\right)\right|\right)^{\text {smooth }} .
$$

\section{Lemma 12:}

The complex $H R\left(\mathbb{Q}_{X}^{\text {per }}\right)^{\text {smooth }}$ consists of c-soft sheaves.

Proof: As noted already in the proof of Lemma 9 we have

$$
\begin{aligned}
H R\left(\mathbb{Q}_{X}^{\text {per }}\right)_{r} & =\underset{n}{\lim } N\left(\operatorname{Sin} \operatorname{Map}\left(.,\left|D K\left(\mathbb{Q}^{\text {per }}\right)\right|^{n}\right)\right)[n]_{r} \\
& =\underset{n}{\lim } \operatorname{Map}^{d}\left(., \operatorname{Map}_{*}\left(\Delta^{n+r} / \Lambda^{n+r},\left|D K\left(\mathbb{Q}^{\text {per }}\right)\right|^{n}\right)\right)
\end{aligned}
$$

The transition maps of the first inductive system come from the maps

$$
\begin{aligned}
N\left(\operatorname{Sin} \operatorname{Map}\left(U, Z^{n}\right)\right) \longrightarrow & N\left(\operatorname{Sin} \operatorname{Map}\left(U, \Omega Z^{n+1}\right)\right) \\
& =\omega N\left(\operatorname{Sin} \operatorname{Map}\left(U, Z^{n+1}\right)\right) \\
& \subseteq N\left(\operatorname{Sin} \operatorname{Map}\left(U, Z^{n+1}\right)\right)[1]
\end{aligned}
$$

which are induced by the structure maps $Z^{n} \longrightarrow \Omega Z^{n+1}$ of the spectrum $Z^{n}:=$ $\left|D K\left(\mathbb{Q}^{\text {per }}\right)\right|^{n}$. As explained in the appendix these latter structure maps and consequently the transition maps are injective. This implies that the functor (.) ${ }^{\text {smooth }}$ commutes with this inductive limit. We obtain

$$
H R\left(\mathbb{Q}_{X}^{\text {per }}\right)_{r}^{\text {smooth }}=\underset{n}{\lim } \operatorname{Map}^{d}\left(., \operatorname{Map}_{*}\left(\Delta^{n+r} / \Lambda^{n+r},\left|D K\left(\mathbb{Q}^{\text {per }}\right)\right|^{n}\right)\right)^{\text {smooth }}
$$

Therefore, by C), $H R\left(\mathbb{Q}_{X}^{\text {per }}\right)_{r}^{\text {smooth }}$ is $c$-soft as an inductive limit of $c$-soft sheaves.

\section{Lemma 13:}

$\mathrm{res}^{\mathrm{smooth}}$ is a quasi-isomorphism. 
Proof: Using again the abbreviation $Z^{n}:=\left|D K\left(\mathbb{Q}^{\text {per }}\right)\right|^{n}$ we compute

$$
\begin{aligned}
& h_{*}\left(\underset{x \in U}{\lim } H R\left(\mathbb{Q}_{X}^{\text {per }}\right)^{\operatorname{smooth}}(U)\right) \\
& =h_{*}\left(\underset{n}{\lim } \underset{x \in U}{\lim } \operatorname{Map}^{d}\left(U, \operatorname{Map}_{*}\left(\Delta^{n+\cdot} / \Lambda^{n+\cdot}, Z^{n}\right)\right)^{\text {smooth }}\right) \\
& =h_{*}\left(\underset{n}{\lim } \underset{(U, H) \in \mathfrak{U}(x)}{\lim } \operatorname{Map}^{d}\left(U, \operatorname{Map}_{*}\left(\Delta^{n+\cdot} / \Lambda^{n+\cdot}, Z^{n}\right)\right)^{H}\right)
\end{aligned}
$$

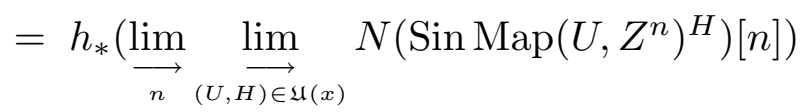

$$
\begin{aligned}
& =\underset{n}{\lim } \underset{(U, H) \in \mathfrak{U}(x)}{\lim } h_{*+n}\left(N\left(\operatorname{Sin} \operatorname{Map}\left(U, Z^{n}\right)^{H}\right)\right) \\
& =\underset{n}{\lim } \underset{(U, H) \in \mathfrak{U}(x)}{\lim } \pi_{*+n}\left(\operatorname{Map}\left(U, Z^{n}\right)^{H}\right) \\
& \left.=\underset{n}{\lim } \pi_{*+n}\left(Z^{n}\right)=\pi_{*}(Z)=h_{*} \underset{x \in U}{\left(\lim _{x \in}\right.} \mathbb{Q}_{X}^{\text {per }}(U)\right) .
\end{aligned}
$$

The first identity in the last line is a consequence of Lemma 11.

Clearly, for any spectrum $Z$ of pointed topological spaces,

$$
\operatorname{Map}_{G}(X, Z):=\operatorname{Map}(X, Z)^{G}:=\left(\operatorname{Map}\left(X, Z^{n}\right)^{G}\right)_{n}
$$

is a subspectrum of $\operatorname{Map}(X, Z)^{\text {smooth }}$. Using the fact that the transition maps of the inductive system which defines $H R\left(\mathbb{Q}_{X}^{\text {per }}\right)$ are injective the computation in Remark 7 gives the following fact.

D) For a compact space $X$ we have

$$
h_{*}\left(H R\left(\mathbb{Q}_{X}^{\text {per }}\right)^{\text {smooth }}(X)^{G}\right)=\pi_{*}\left(\operatorname{Map}\left(X,\left|D K\left(\mathbb{Q}^{\text {per }}\right)\right|\right)^{G}\right) .
$$

\section{Proposition 14:}

If $G$ and $X$ are compact then the map res ${ }^{\text {smooth }}$ induces isomorphisms

$$
\underset{j \in \mathbf{Z}}{\oplus} H^{*+2 j}(X, \mathbb{Q})^{G} \stackrel{\cong}{\longrightarrow} \pi_{*}\left(\operatorname{Map}\left(X,\left|D K\left(\mathbb{Q}^{\text {per }}\right)\right|\right)^{G}\right)
$$

and

$$
\underset{j \in \mathbf{Z}}{\oplus} H_{G, c}^{*+2 j}(p t, X) \stackrel{\cong}{\longrightarrow} \pi_{*}\left(\operatorname{Map}\left(\hat{X},\left|D K\left(\mathbb{Q}^{\text {per }}\right)\right|\right)^{G}\right) \underset{\mathbb{Q}}{\otimes} \mathbb{C}
$$


Proof: Since the functor $(.)^{\infty}$ is left exact and commutes with arbitrary direct sums we can argue, using C), exactly as in the proof of Prop. 10 and obtain the isomorphism

$$
\underset{j \in \mathbf{Z}}{\oplus} H^{*+2 j}(X, \mathbb{Q}) \stackrel{\cong}{\longrightarrow} h_{*}\left(H R\left(\mathbb{Q}_{X}^{\text {per }}\right) \cdot{ }^{\operatorname{smooth}}(X)\right)
$$

We then pass to $G$-fixed vectors which, by the compactness of $G$, is exact. Together with D) this gives the first isomorphism in the assertion. The second one is obtained from the first one (for $\hat{X}$ ) by passing to the tensor product with $\mathbb{C}$ and using

$$
H^{*}(\hat{X}, \mathbb{Q})^{G} \underset{\mathbb{Q}}{\otimes} \mathbb{C}=\left(H^{*}(\hat{X}, \mathbb{Q}) \underset{\mathbb{Q}}{\otimes} \mathbb{C}\right)^{G}=H^{*}(\hat{X}, \mathbb{C})^{G}
$$

(compare [KS] II.2.6.6).

This last result should be compared with the Atiyah-Segal-Jänich theorem ([Ati], [Se3],[Jän]) which says that, for compact $G$ and $X$, we have

$$
K_{G}^{*}(X)=\pi_{*}\left(\operatorname{Map}(X, \mathbf{K})^{G}\right)
$$

where $\mathbf{K}$ is an appropriate version of the $K$-theory spectrum of the point. One can in fact show that the cohomological Chern character in section 2 lifts to a "stable" map of spectra

$$
\operatorname{Map}(X, \mathbf{K})^{G} \longrightarrow \operatorname{Map}\left(\hat{X},\left|D K\left(\tilde{\mathbb{Q}}^{\text {per }}\right)\right|\right)^{G} .
$$

The careful reader must have noticed that in section 2 we took $\mathbb{C}$ as the field of coefficients since we needed the roots of unity in it whereas in the present section we worked over $\mathbb{Q}$ because we needed the field of coefficients to be countable. One way to reconcile these two requirements is to always work over the field $\tilde{\mathbb{Q}}$ arising by adjoining all roots of unity to $\mathbb{Q}$.

\section{Appendix: The Dold-Kan correspondence}

Let $\mathfrak{A}$ be an abelian category. By a complex, resp. a chain complex, in $\mathfrak{A}$ we mean an in both directions unbounded complex, resp. a complex concentrated in nonnegative degrees, of homological type in $\mathfrak{A}$. There are quasi-inverse equivalences of categories

$$
\text { simplicial objects in } \mathfrak{A} \underset{D K}{\stackrel{N}{\longrightarrow}} \text { chain complexes in } \mathfrak{A}
$$


given by the normalization functor $N$ and the Dold-Kan functor $D K$ as described, for example, in [Wei] 8.4 ( $D K$ there is called $K)$. If $A$. is a simplicial object in $\mathfrak{A}$ then the $m$-th object $N(A .)_{m}$ of the chain complex $N(A$.) is given as the simultaneous kernel of all but the last face map from $A_{m}$; the last face map becomes the differential of the complex. All we need to know about the functor $D K$ is that, for any chain complex $C$., the $m$-th object $D K(C .)_{m}$ in the simplicial object $D K(C$. $)$ is a finite direct sum of objects $C_{k}$ for $k \leq m$. Any additive functor between abelian categories induces functors on both sides of the above equivalence which are compatible with $N$ and $D K$.

We are mainly interested in two kinds of abelian categories. One is the category of $\mathbb{Q}$-vector spaces (or $K$-vector spaces for some fixed countable field $K$ ). Any simplicial $\mathbb{Q}$-vector space $A$. in particular is a pointed (by the trivial vector space) simplicial set; its topological realization

$$
|A .|=\left(\bigcup_{m \geq 0} \Delta^{m} \times A_{m}\right) / \sim
$$

(where $\sim$ is a certain equivalence relation) is a pointed Hausdorff space. One has the natural isomorphisms

$$
\pi_{*}(|A .|)=h_{*}(N A .)
$$

and, for any chain complex of $\mathbb{Q}$-vector spaces $C$. ,

$$
\pi_{*}(|D K(C .)|)=h_{*}(C .) .
$$

The other abelian category is the category of sheaves of $\mathbb{Q}$-vector spaces (or $K$ vector spaces) on some Hausdorff space $X$. As pointed out the functor of taking sections in an open subset $U \subseteq X$ commutes with $D K$, i.e., we have

$$
D K(\mathcal{S} .)(U):=D K(\mathcal{S} .(U))
$$

for any chain complex of sheaves $\mathcal{S}$. . By $\mid D K(\mathcal{S}$.)| we denote the presheaf of pointed Hausdorff spaces on $X$ defined by

$$
|D K(\mathcal{S} .)|(U):=|D K(\mathcal{S} .(U))| .
$$

The loop functor $\omega$ on chain complexes in $\mathfrak{A}$ is defined by

$$
\omega C .:=\left[\ldots \longrightarrow C_{3} \longrightarrow C_{2} \longrightarrow \operatorname{ker}\left(d_{1}\right)\right]
$$

where the last term $\operatorname{ker}\left(d_{1}\right)$ is put in degree 0 . An $\omega$-spectrum of chain complexes in $\mathfrak{A}$ is a collection of chain complexes $C .^{(n)}$ together with isomorphisms of 
complexes $C \cdot{ }^{(n)} \stackrel{\cong}{\longrightarrow} \omega C \cdot{ }^{(n+1)}$. For any $n \in \mathbb{Z}$ the truncation functor $t_{\geq n}$ on complexes in $\mathfrak{A}$ is defined by

$$
t_{\geq n} \text { D. }:=\left[\ldots \longrightarrow D_{n+2} \longrightarrow D_{n+1} \longrightarrow \operatorname{ker}\left(d_{n}\right) \longrightarrow 0 \longrightarrow \ldots\right]
$$

where degrees are unchanged. The chain complexes $D .{ }^{(n)}:=\left(t_{\geq-n} D.\right)[-n]$ satisfy $D .^{(n)}=\omega D .^{(n+1)}$ and hence form an $\omega$-spectrum of chain complexes. It is easy to see that the functor

$$
\begin{aligned}
\text { complexes in } \mathfrak{A} & \stackrel{\sim}{\longrightarrow} \begin{array}{l}
\omega \text {-spectra of chain } \\
\text { complexes in } \mathfrak{A}
\end{array} \\
D . & \longmapsto\left(D \cdot{ }^{(n)}\right)_{n}
\end{aligned}
$$

is an equivalence of categories.

On the other hand let $S$. be a pointed simplicial set. We define a new simplicial set $\omega S$. by

$$
(\omega S)_{m}:=\left\{s \in S_{m+1}: d_{0}=p t, d_{1} \ldots d_{m+1}=p t\right\}
$$

with the face and degeneracy maps induced by those of $S$. omitting the 0 -th ones. This is the "small" loop space functor on pointed simplicial sets. It is clear from the definition that this functor respects simplicial $\mathbb{Q}$-vector spaces and that it can be defined correspondingly on simplicial objects in the abelian category $\mathfrak{A}$. Similarly to the case of chain complexes we have the obvious notions of an $\omega$ spectrum of pointed simplicial sets and of an $\omega$-spectrum of simplicial objects in $\mathfrak{A}$. Since the normalization functor $N$ (and hence the functor $D K$ ) clearly is compatible with the functors $\omega$ on both sides the Dold-Kan correspondence extends to an equivalence of categories

$$
\begin{aligned}
D K: \text { complexes in } \mathfrak{A} & \stackrel{\sim}{\longrightarrow} \begin{array}{l}
\text { w-spectra of simplicial } \\
\text { objects in } \mathfrak{A}
\end{array} \\
D . & \longmapsto\left(D K\left(D \cdot \cdot^{(n)}\right)\right)_{n}
\end{aligned}
$$

with quasi-inverse $N\left(\left(A .^{(n)}\right)_{n}\right):=\underset{n \geq 0}{\lim } N\left(A \cdot^{(n)}\right)[n]$.

The functor $\omega$ on pointed simplicial sets has a left adjoint $\Sigma$ called the Kan suspension (compare [Kan] $\S 2$ and [Jar] §4.4). According to [Kan] 2.3 there is a natural isomorphism

$$
|\Sigma S .| \cong S|S .|
$$

Hence any $\omega$-spectrum of pointed simplicial sets $\left(S \cdot{ }^{(n)}\right)_{n}$ has a topological realization

$$
\left|\left(S .^{(n)}\right)_{n}\right|:=\left(\left|S \cdot{ }^{(n)}\right|\right)_{n}
$$


which is a spectrum of pointed topological spaces. In terms of the loop space functors the structure maps of the topological realization arise from the natural map

$$
|\omega S .| \stackrel{\text { adj }}{\longrightarrow} \Omega S|\omega S .| \cong \Omega|\Sigma \omega S .| \stackrel{\Omega \mid \text { adj } \mid}{\longrightarrow} \Omega|S .| .
$$

We emphasize that this map is injective. This is clear for the left adjunction map. For the right map this follows from the injectivity of the adjunction map $\Sigma \omega S . \longrightarrow S .([\mathrm{Kan}] 6.3)$ and the fact that the functors $\Omega$ and $|$.$| preserve injective$ maps ([GZ] III.3.2). This means that for an $\omega$-spectrum of pointed simplicial sets $\left(S .^{(n)}\right)_{n}$ the structure maps of its topological realization

$$
\left|S .^{(n)}\right| \hookrightarrow \Omega\left|S .^{(n+1)}\right|
$$

are injective (as well as weak equivalences). For a complex of abelian groups $D$. we obtain

$$
\begin{aligned}
\pi_{r}(|D K(D .)|) & =\underset{n>-r}{\lim } \pi_{n+r}\left(\left|D K\left(D \cdot{ }^{(n)}\right)\right|\right)=\underset{n>-r}{\lim } h_{n+r}\left(D \cdot .^{(n)}\right) \\
& =\underset{n>-r}{\lim _{n}} h_{r}\left(t_{\geq-n} D .\right)=h_{r}(D .) .
\end{aligned}
$$

Since the functors $\omega$ and $t_{\geq n}$ are limits they sheafify in the most direct way by performing them sectionwise (without subsequent sheafification!). We therefore have (again by sectionwise definition) the equivalence of categories

$$
\begin{array}{lll}
\text { complexes of sheaves of } & \stackrel{D K}{\longrightarrow} & \omega \text {-spectra of simplicial sheaves } \\
\mathbb{Q} \text {-vector spaces on } X & \longleftarrow & \text { of } \mathbb{Q} \text {-vector spaces on } X
\end{array}
$$

and the topological realization functor

$\omega$-spectra of simplicial sheaves of $\mathbb{Q}$-vector spaces on $X$

$$
\downarrow|\cdot|
$$

presheaves of spectra of pointed topological spaces on $X$. 


\section{References}

[Atiy] Atiyah M.F.: K-theory. Benjamin 1967

[Ati] Atiyah M.F.: Bott periodicity and the index of elliptic operators. Quart. J. Math. Oxford 19, 113-140 (1968)

[BC] Baum P., Connes A.: Chern character for discrete groups. In A fete of topology, pp. 163-232. Academic Press 1988

[BCH] Baum P., Connes A., Higson N.: Classifying Space for Proper Actions and $K$-Theory of Group $C^{*}$-Algebras. In $C^{*}$-Algebras: 1943-1993 (Ed. Doran). Contemporary Math. 167, pp. 241-291. AMS 1994

[BZ] Bernstein J., Zelevinskii A.: Representations of the group $G L(n, F)$ where $F$ is a non-archimedean local field. Russian Math. Surveys 31, 1-68 (1976)

[B-GT] Bourbaki N.: General Topology. Springer 1989

[Bre] Bredon G.: Equivariant Cohomology Theories. Lect. Notes in Math. 34. Springer 1967

[GZ] Gabriel P., Zisman M.: Calculus of Fractions and Homotopy Theory. Springer 1967

[Har] Hartshorne R.: Residues and Duality. Lect. Notes in Math. 20. Springer 1966

[Hub] Huber P.: Homotopical Cohomology and Čech Cohomology. Math. Ann. 144, 73-76 (1961)

[Jän] Jänich K.: Vektorraumbündel und der Raum der Fredholm-Operatoren. Math.Ann. 161, 129-142 (1965)

[Jar] Jardine J. F.: Generalized Etale Cohomology Theories. Birkhäuser 1997

[Kan] Kan D.: Semisimplicial spectra. Illinois J. Math. 7, 463-478 (1963)

[Kar] Karoubi M.: K-Theory. Springer 1978

[KS] Kashiwara M., Schapira P.: Sheaves on Manifolds. Springer 1990

[Ka1] Kasparov G.: The operator $K$-functor and extensions of algebras. Math. USSR Izv. 16, 513-572 (1981)

[Ka2] Kasparov G.: Equivariant $K K$-theory and the Novikov conjecture. Invent. math. 91, 147-201 (1988)

[LW] Lundell A., Weingram S.: The topology of CW complexes. Van Nostrand 1969

[McL] MacLane S.: Homology. Springer 1975 
[Mas] Masuda T.: Bivariant homology-cohomology theory and Chern character on $K K$-theory. In Geometric methods in operator algebras (Kyoto 1983). Pitman Research Notes 1986

[Mil] Milnor J.: On spaces having the homotopy type of a CW-complex. Transact. AMS 90, 272-280 (1959)

[Rha] de Rham G.: Differentiable Manifolds. Springer 1984

[Sch] Schneider P.: Equivariant homology for totally disconnected groups. J. Algebra 203, 50-68 (1998)

[Seg] Segal G.: Equivariant K-theory. Publ. Math. IHES 34, 129-151 (1968)

[Se2] Segal G.: The representation ring of a compact Lie group. Publ. Math. IHES 34, 113-128 (1968)

[Se3] Segal G.: Fredholm complexes. Quart. J. Math. Oxford 21, 385-402 (1970)

[Slo] Słominska J.: On the Equivariant Chern Homomorphism. Bull. l'Acad. Pol. Sci. 24, 909-913 (1976)

[Spa] Spanier E.: Algebraic Topology. McGraw-Hill 1966

[Wei] Weibel C. A.: An introduction to homological algebra. Cambridge Univ. Press 1994

Peter Schneider

Mathematisches Institut

Westfälische Wilhelms-Universität Münster

Einsteinstr. 62

D-48149 Münster, Germany

pschnei@math.uni-muenster.de

http://www.uni-muenster.de/math/u/schneider

Paul Baum

Mathematics Department

McAllister Building

The Pennsylvania State University

University Park, PA 16802

baum@math.psu.edu

http://www.math.psu.edu/baum/ 